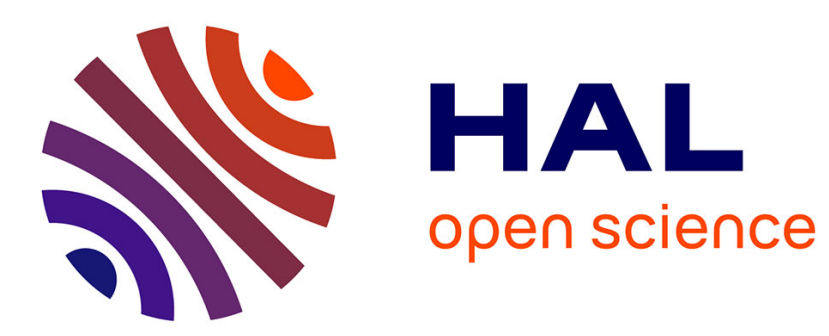

\title{
Upscaling Reactive Transport Under Hydrodynamic Slip Conditions in Homogeneous Porous Media
}

\author{
Francisco J Valdés-Parada, Didier Lasseux, Stephen Whitaker
}

\section{To cite this version:}

Francisco J Valdés-Parada, Didier Lasseux, Stephen Whitaker. Upscaling Reactive Transport Under Hydrodynamic Slip Conditions in Homogeneous Porous Media. Water Resources Research, 2020, 56 (1), 10.1029/2019WR025954 . hal-03151320

\author{
HAL Id: hal-03151320 \\ https://hal.science/hal-03151320
}

Submitted on 24 Feb 2021

HAL is a multi-disciplinary open access archive for the deposit and dissemination of scientific research documents, whether they are published or not. The documents may come from teaching and research institutions in France or abroad, or from public or private research centers.
L'archive ouverte pluridisciplinaire HAL, est destinée au dépôt et à la diffusion de documents scientifiques de niveau recherche, publiés ou non, émanant des établissements d'enseignement et de recherche français ou étrangers, des laboratoires publics ou privés. 


\section{Water Resources Research}

\section{DOI: $10.1029 / 2019$ WR025954}

Key Points:

- The influence of hydrodynamic slip on macroscopic reactive transport is analyzed

- The conditions for the total dispersion tensor and effective convective velocity to be reaction-independent are determined - The macroscopic model is validated by comparison with pore-scale direct numerical simulations

Correspondence to:

D. Lasseux,

didier.lasseux@u-bordeaux.fr

\section{Citation:}

Valdes-Parada, F. J., Lasseux, D., \& Whitaker, S. (2020). Upscaling reactive transport under hydrodynamic slip conditions in homogeneous porous media. Water Resources Research, 56, e2019WR025954. https://doi.org/10. 1029/2019WR025954

\section{Upscaling Reactive Transport Under Hydrodynamic Slip Conditions in Homogeneous Porous Media}

\author{
Francisco J. Valdés-Parada ${ }^{1}$, Didier Lasseux ${ }^{2}$, and Stephen Whitaker ${ }^{3}$ \\ ${ }^{1}$ Área de Ingeniería en Recursos Energéticos, Universidad Autónoma Metropolitana-Iztapalapa, Mexico City, Mexico, \\ ${ }^{2}$ CNRS, UMR 5295, Univ. Bordeaux, Talence, France, ${ }^{3}$ Department of Chemical Engineering, University of California at \\ Davis, Davis, CA, USA
}

\begin{abstract}
Reactive transport of a dilute species in a Newtonian fluid saturating a homogeneous porous medium for slip flow is analyzed in this work. Incompressible Newtonian flow with a first-order (Navier) slip boundary condition is considered together with a first-order heterogeneous reaction. Kinetic numbers range up to unity while Knudsen numbers, characteristic of slip-flow, are smaller than approximately 0.1 . The pore-scale problem is upscaled, using the volume averaging method, to obtain a macroscopic transport equation (referred to as the complete upscaled model) operating at the Darcy scale. Using order of magnitude estimates and an expansion in terms of the Kinetic number, simplifications in the expressions of the effective coefficients are explored. The conditions under which the effective convective velocity and the total dispersion tensor are independent of reaction are derived. Under such conditions, a simplified version of the macroscopic transport equation is obtained. This is referred to as the simplified model. Numerical results for a simple structure indicate that the longitudinal dispersion coefficient decreases with decreasing Knudsen number, while the opposite holds for the transverse component of the total dispersion tensor, the effective convective velocity, and the effective reaction rate coefficient. These effects are more evident when the Péclet number increases (i.e., in the convective-favored regime) and when slip effects are more pronounced. Results predicted by the simplified and the complete versions of the upscaled model are validated with direct numerical simulations of the pore-scale problem on a two-dimensional model structure.
\end{abstract}

\section{Introduction}

The study of reactive transport in porous media has been long-reported in the literature due to its wide range of applications that may range from chemical reactor engineering (Froment et al., 2010) to large-scale soil remediation processes (Bear, 2018). In the past decade, this transport phenomenon has attracted renewed interest due to the key role that it plays in environmental applications such as bio-remediation (Oya \& Valocchi, 1998) and carbon dioxide sequestration (see, for instance, Niemi et al., 2017), among others. In this particular application, there are several studies focused on reactive transport based either on pore-scale modeling (Tian \& Wang, 2017) or on the use of Darcy-scale models (Nordbotten \& Celia, 2011). For example, Gao et al. (2017) used a Lattice-Boltzmann approach to study the effects of unsteady local reactions and unstable dissolution fronts, thus enabling the assessment of porosity changes due to reaction fronts. On the same line of research, Bringedal et al. (2016) derived a macroscale model using the homogenization technique for a situation in which ions dissolved in the fluid precipitate on the porous skeleton while the solid grain minerals dissolve in the fluid saturating the pores.

Despite the remarkable advances in pore-scale modeling (e.g., Alhashmi et al., 2015; Scheibe et al., 2015; Yang et al., 2016), the use of Darcy-scale models is still an active research field, since it allows making large-scale predictions at a somewhat reasonable computational cost. However, Darcy-scale models are limited by the corresponding set of length-scale constraints and assumptions implicit in their derivation. During the past decade, there have been many works focused on identifying the threshold of application of these types of models for diffusive conditions (Battiato et al., 2009), diffusive-convective conditions considering linear (Boso \& Battiato, 2013) and nonlinear (Battiato \& Tartakovsky, 2011) reaction kinetics. In the work by Boso and Battiato (2013), it was emphasized that heterogeneous reaction leads to more stringent constraints than in situations where the reaction is homogeneous. 
The derivation of upscaled models can be carried out by a number of available techniques such as homogenization (Auriault et al., 2009), volume averaging (Whitaker, 1999b), or the thermodynamically constrained averaging theory (Gray \& Miller, 2014). The first two approaches have been largely used for the study of reactive transport and were shown to be equivalent (Davit et al., 2013). In these methods, the macroscale model is derived by spatially smoothing the governing equations at the pore scale. The resulting model is written in terms of effective-medium coefficients that can be predicted by solving ancillary closure problems in representative zones of the pore-scale structure. These representative zones are usually chosen to be periodic unit cells. Recently, Yan and $\mathrm{Li}$ (2017) used volume averaging to compute the dispersion coefficient in many different types of unit cells. They noticed that the scattering of dispersion coefficients decreases by increasing the unit cell size. In addition, their modeling predictions were validated with existing correlations and experimental data. During the same period, Porta et al. (2016) used the volume averaging method to study reactive mass transport under unsteady conditions, applicable even at the closure-problem level, and compared their results with other available macroscale models that require calibration with experimental data. This analysis followed a previous work by Porta et al. (2013) where it was argued that the resulting macroscale model, which is nonlocal in time, can be approximated by its local counterpart since quasi-steady conditions are obtained for very short characteristic times at the closure level.

In most of the above references, transport coefficients involved in the upscaled model have been assumed to be independent of the reaction rate despite the long debate in the literature on this dependency (e.g., Dadvar \& Sahimi, 2007; Zhang \& Seaton, 1994). Under a volume averaging framework, it was shown that the dispersion coefficient is, in general, dependent on the reaction rate (Valdés-Parada et al., 2011). Results obtained in this work confirmed those reported earlier by Edwards et al. (1993) indicating that the dispersion coefficient decreases as the Kinetic number increases. A more systematic analysis in the limit cases of large and small Péclet and Kinetic numbers was proposed by Mauri (1991). Under nonconvective conditions, a recent work by Valdés-Parada et al. (2017) was carried out to revisit the derivation of the upscaled model in order to investigate the impact of reaction on the effective diffusivity. When the Kinetic number remains smaller than unity (a situation of practical use for the catalyst to be efficient; Froment et al., 2010), the dependence of the effective diffusivity on the reaction rate was found to be negligible in the prediction of the macroscopic concentration dynamics.

To the best of our knowledge, when convective mass transport is involved, the impact of hydrodynamic slip on the effective dispersion and reaction coefficients has not been documented so far. The analysis of dispersion in the presence of slip flow was performed in capillary tubes and beds of spheres for applications in chromatography (Adrover et al., 2009; Beauchamp \& Schure, 2019; Smits et al., 2014; Yan \& Wang, 2013) in order to investigate the effect of slip on the plate height. However, the effect of reaction was not explicitly taken into account in these references. One should note the work by Altevogt et al. (2003) in which the dispersion problem involving a slip effect was studied in a homogeneous porous medium using the volume averaging method. In this reference, no reaction was considered and slip was assumed to be induced by mass and not by momentum transport. However, there are many situations for which hydrodynamic slip effects are of special interest. This is the case when the dispersion-reaction process takes place in a gas-saturated porous medium and when the Knudsen number is not exceedingly small compared to 1 . The objective of the present work is to address this problem. Meanwhile, the effect of the potential coupling between dispersive and reactive mechanisms on the total dispersion coefficient and effective convective velocity is revisited within the context of slip flow.

The analysis carried out in this work is organized as follows. In section 2, the pore-scale model is presented, considering incompressible Newtonian flow with a first-order (Navier) slip condition for a fluid (gas) saturating a homogeneous porous material. A chemical species is supposed to be transported by convection and Fickian diffusion while experiencing a first-order heterogeneous reaction. In order to obtain a macroscopic model operating at the Darcy scale, an upscaling approach is followed using the volume averaging method, which is briefly summarized in section 3. The application of this upscaling technique to the pore-scale problem, under time and length-scale constraints that are recalled, is reported in section 4 . This yields a macroscopic model for which simplifications are investigated in section 5. These simplifications are based on order of magnitude estimates, on the one hand, and on an expansion in terms of the Kinetic number, which is supposed to remain smaller than unity, on the other hand. The relevance of these simplifications is further analyzed in section 6. Predictions of the effective coefficients, namely, the effective convective velocity, longitudinal and transverse total dispersion, and the effective reaction rate coefficient, are reported in 


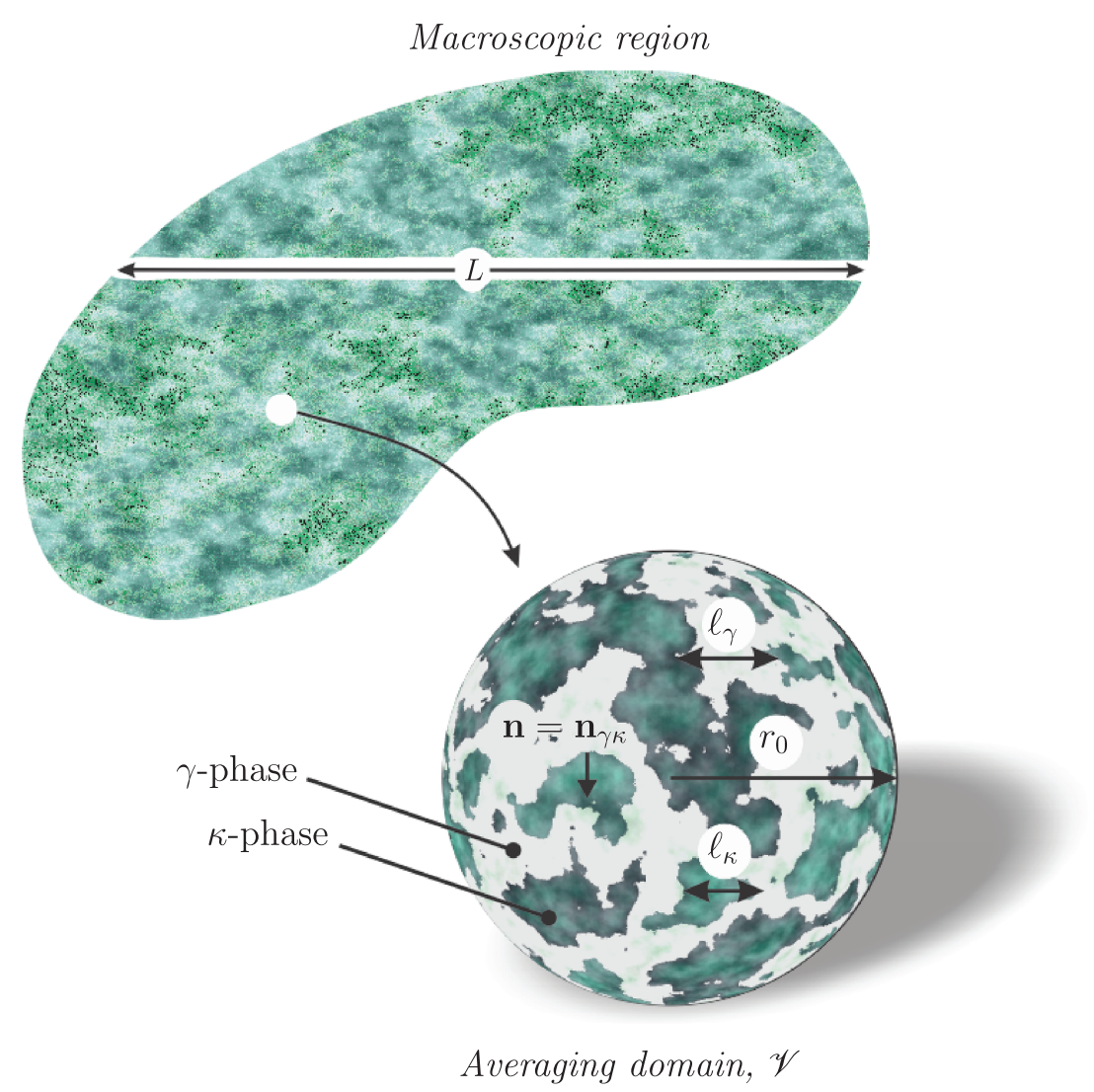

Figure 1. Sketch of a porous medium system (macroscopic region) composed of a fluid ( $\gamma$-phase) and a solid ( $\kappa$-phase) including the averaging domain $\mathscr{V}$, the characteristic lengths, and the unit normal vector $\mathbf{n}$.

section 6.1 for both the complete and simplified versions of the macroscopic model. The simplified upscaled model is validated in section 6.2 by comparison with pore-scale direct numerical simulation results on a simple 2D structure. Finally, conclusions are drawn in section 7.

\section{Pore-Scale Model}

The process under consideration consists of convective and diffusive mass transport of a dilute chemical species (species $A$ ) in a rigid and homogeneous porous medium such as the one illustrated in Figure 1. Here, the fluid phase is denoted as the $\gamma$-phase and the solid phase is the $\kappa$-phase. Assuming steady, incompressible flow of a Newtonian fluid, the problem can be described by the following pore-scale governing equations:

$$
\nabla \cdot \mathbf{v}=0, \quad \text { in the } \gamma \text {-phase }
$$

$$
\begin{gathered}
\mathbf{0}=-\nabla p+\rho \mathbf{g}+\mu \nabla^{2} \mathbf{v}, \quad \text { in the } \gamma \text {-phase, } \\
\frac{\partial c}{\partial t}+\nabla \cdot(c \mathbf{v})=\nabla \cdot(\mathscr{D} \nabla c), \quad \text { in the } \gamma \text {-phase. }
\end{gathered}
$$

Here, $p$ and $\mathbf{v}$ represent the pressure and velocity of the fluid phase that is assumed to have a constant density $\rho$ and dynamic viscosity $\mu$. In addition, $c$ denotes the molar concentration of $\operatorname{species} A$ that is transported by convection and diffusion within the fluid phase, and $\mathscr{D}$ represents the mixture diffusivity of species $A$. This coefficient is considered to be a known constant. Inertia is assumed to be negligible in the momentum equation (1b) with the idea that the Reynolds number, $R e=\rho v \ell_{\gamma} / \mu$, is such that $R e \ll 1, \ell_{\gamma}$ and $v$ representing the characteristic pore diameter and the order of magnitude of the fluid velocity, respectively. The constraints supporting the incompressibility assumption are $M a^{2} \ll 1$ and $M a \ll F r, M a=v / C$ and $\mathrm{Fr}=v / \sqrt{\mathrm{g} \ell_{\gamma}}$ being respectively the Mach and Froude numbers of the flow; $C$ is the speed of sound 
(Whitaker, 1988, 1999a). Furthermore, steady flow is subject to the time constraint $t \gg \operatorname{Re}_{\gamma} / \nu$. Note that this time scale must be considered as being uncorrelated to mass transport. This means that transport of species $A$ starts after steady flow is established. Equation (1c) is written in a form derived in Whitaker (2009) (see equations (164) and (173) therein). The validity of this equation is subject to two restrictions, namely, (i) a constant total molar concentration within the fluid phase and (ii) the transported species $A$ is dilute.

In this work, the interest is focused on the case where the mean-free path of the fluid molecules $(\lambda)$ is not exceedingly small compared to the characteristic pore diameter $\left(\ell_{\gamma}\right)$, so that Knudsen effects are significant and flow can be considered to be in the slip regime (Harley et al., 1995). This means that the Knudsen number, $K n=\lambda / \ell_{\gamma}$, remains smaller than $\sim 0.1$. Under these circumstances, the interfacial boundary condition may be stated as the classical first-order slip condition (Maxwell, 1879)

$$
\mathbf{v}=-\xi \lambda(\mathrm{I}-\mathbf{n n}) \cdot\left[\mathbf{n} \cdot \nabla \mathbf{v}+\nabla \mathbf{v}^{T}\right], \quad \text { at the } \gamma-\kappa \text { interface. }
$$

Here, $\xi$ is a parameter related to the tangential momentum accommodation coefficient (Arkilic et al., 2001) and is usually close to 1 in practice. In addition, $\mathbf{I}$ and $\mathbf{n}$ represent the identity tensor and the unit normal vector directed from the fluid phase towards the solid phase, respectively. For mass transport, it is assumed that a heterogeneous first-order chemical reaction, characterized by a constant reaction rate coefficient, $k$, takes place at the solid-fluid interface, so that the normal diffusive flux of species $A$ can be expressed as

$$
-\mathbf{n} \cdot(\mathscr{D} \nabla c)=k c, \quad \text { at the } \gamma-\kappa \text { interface. }
$$

The pore-scale model is completed with the corresponding boundary conditions at the macroscopic entrances and exits, as well as by the initial condition for the species concentration. However, this information is not necessary for the derivation of the macroscale model and it is not specified here for the sake of brevity.

\section{Generalities on Upscaling by Means of the Volume Averaging Method}

The method of volume averaging is an upscaling approach that allows deriving macroscale models by spatially smoothing the microscale heterogeneities in hierarchical systems (Whitaker, 1999b). This approach has two salient features. First, it allows one to clearly identify the time- and length-scale constraints together with assumptions under which the macroscale model is applicable. Second, it includes a closure scheme in order to predict the effective-medium coefficients involved in the resulting upscaled model. The purpose of this section is to present the basic elements of the volume averaging method, which are used to derive the macroscopic equations for momentum and mass transport.

For a multiscale system such as the one sketched in Figure 1, a separation of several orders of magnitude between the characteristic length of the macroscale, $L$, and those associated to the microscale, $\ell_{\gamma}, \ell_{\kappa}$ is assumed. Here, $\ell_{K}$ is the characteristic length associated to the solid phase. In between these length scales, it is further assumed that a support scale, $r_{0}$, that characterizes the size of the averaging domain, $\mathscr{V}$, can be defined such that

$$
\ell_{\gamma}, \ell_{\kappa} \ll r_{0} \ll L
$$

According to this scale hierarchy, the averaging domain can be considered to be representative. This means that it allows one to filter out the redundant information from the microscale although it is small enough compared to $L$ to keep sufficient information at the scale $r_{0}$. On this basis, the superficial and intrinsic averaging operators of a piecewise continuous function, $\psi$, defined in the $\gamma$-phase, can be respectively defined as follows:

$$
\begin{aligned}
\langle\psi\rangle & =\frac{1}{V} \int_{\mathscr{V}_{\gamma}} \psi d V, \\
\langle\psi\rangle^{\gamma} & =\frac{1}{V_{\gamma}} \int_{\mathscr{V}_{\gamma}} \psi d V .
\end{aligned}
$$



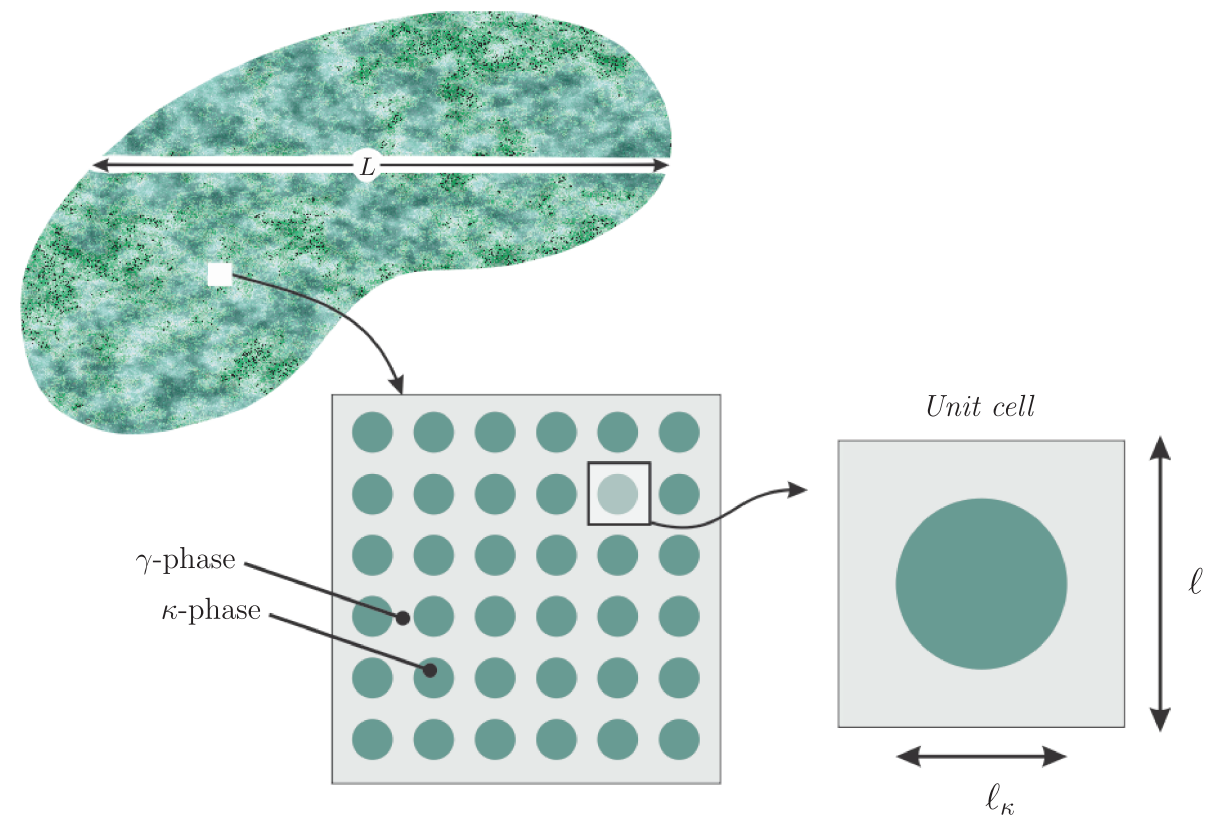

Figure 2. Sketch of a simple 2D periodic representation of the pore-scale geometry and unit cell for the closure problems solution.

Here, $V$ is the volume of the averaging domain, $\mathscr{V}_{\gamma}$ is the domain (of volume $V_{\gamma}$ ) occupied by the fluid phase within $\mathscr{V}$. These two averaging operators are related by means of the Dupuit-Forchheimer equation:

$$
\langle\psi\rangle=\varepsilon\langle\psi\rangle^{\gamma},
$$

$\varepsilon=V_{\gamma} / V$ being the volume fraction of the fluid phase within the averaging domain, that is, the porosity.

Application of the averaging operator to the pore-scale equations requires one to interchange spatial integration with temporal and spatial differentiation. This is carried out by means of the general transport theorem (Slattery, 1999) and the spatial averaging theorem (Howes \& Whitaker, 1985). These are respectively given by

$$
\begin{gathered}
\left\langle\frac{\partial \psi}{\partial t}\right\rangle=\frac{\partial\langle\psi\rangle}{\partial t}-\frac{1}{V} \int_{\mathscr{A}_{\gamma \kappa}} \mathbf{n} \cdot \psi \mathbf{w} d A, \\
\langle\nabla \psi\rangle=\nabla\langle\psi\rangle+\frac{1}{V} \int_{\mathscr{A}_{\gamma \kappa}} \mathbf{n} \psi d A,
\end{gathered}
$$

where $\mathbf{w}$ is a vector that denotes the displacement velocity of the solid-fluid interface. In addition, in most applications of the volume averaging method, it is convenient to spatially decompose pore-scale variables according to Gray (1975):

$$
\psi=\langle\psi\rangle^{\gamma}+\tilde{\psi}
$$

Here, $\tilde{\psi}$ represents the spatial variations of $\psi$ with respect to its intrinsic average. In this way, once $\tilde{\psi}$ is determined by means of a closure problem solution, the resulting model is closed. The solution domain of the closure problems is usually chosen to be a periodic unit cell of characteristic size $\ell$ (see Figure 2). The final model often involves effective-medium coefficients that are defined in terms of integrals of closure variables. Details about the steps involved in the volume averaging method can be found in Whitaker (1999b) and Wood and Valdés-Parada (2013). 


\section{Species Mass Transport and Reaction. The Complete Upscaled Model}

In this section, the methodology described above is used to average the pore-scale problem in equations (1).

The result of applying the intrinsic averaging operator to the species conservation equation, taking into account the averaging theorem, the interfacial boundary conditions, and the spatial decompositions of the concentration and velocity, can be expressed as (see details in Whitaker, 1999b)

$$
\begin{aligned}
& \frac{\partial\langle c\rangle^{\gamma}}{\partial t}+\nabla \cdot\left(\langle c\rangle^{\gamma}\langle\mathbf{v}\rangle^{\gamma}\right)+\nabla \cdot\langle\tilde{c} \mathbf{v}\rangle^{\gamma}=\nabla \cdot\left[\mathscr{D}\left(\nabla\langle c\rangle^{\gamma}+\frac{1}{V_{\gamma}} \int_{\mathscr{A}_{\gamma \kappa}} \mathbf{n} \tilde{c} \quad d A\right]\right. \\
& -\frac{k A_{\gamma \kappa}}{V_{\gamma}}\langle c\rangle^{\gamma}-\frac{k}{V_{\gamma}} \int_{\mathscr{A}_{\gamma \kappa}} \tilde{c} d A .
\end{aligned}
$$

This form of the macroscale transport equation represents the unclosed model that is written in terms of filters of information (i.e., interfacial and volumetric integrals) from the pore scale contained in the spatial concentration deviations. In the above equation, $\langle\mathbf{v}\rangle^{\gamma}$ is the intrinsic average velocity, which is determined from the average flow model. The macroscopic momentum equation in this model is a Darcy-like equation that accounts for slip effects. It was derived by Lasseux et al. (2016), and it is given by

$$
\langle\mathbf{v}\rangle=-\frac{\mathrm{K}_{s}}{\mu} \cdot\left(\nabla\langle p\rangle^{\gamma}-\rho \mathbf{g}\right) .
$$

Here, the apparent permeability tensor, $\mathrm{K}_{s}$, whose symmetry properties were reported by Lasseux and Valdés-Parada (2017), is obtained from the solution of a closure problem which was reported in the literature (see sections 3.1 and 4.1 in Lasseux et al., 2016).

On the basis of the dilute assumption, flow is decoupled from species transport, and, for this reason, the fluid velocity, as well as its average and spatial deviations, can be regarded as known solenoidal fields for the rest of the derivations.

To progress towards a closed form of equation (7), a governing equation for $\tilde{c}$ is required. This is achieved by subtracting equation (7) from equation (1c), yielding

$$
\begin{aligned}
& \frac{\partial \tilde{c}}{\partial t}+\mathbf{v} \cdot \nabla \tilde{c}+\tilde{\mathbf{v}} \cdot \nabla\langle c\rangle^{\gamma}-\nabla \cdot\langle\tilde{c} \mathbf{v}\rangle^{\gamma}=\mathscr{D} \nabla^{2} \tilde{c}-\nabla \cdot\left(\frac{\mathscr{D}}{V_{\gamma}} \int_{\mathscr{A}_{\gamma \kappa}} \mathbf{n} \tilde{c} d A\right) \\
& \quad+\frac{k A_{\gamma \kappa}}{V_{\gamma}}\langle c\rangle^{\gamma}+\frac{k}{V_{\gamma}} \int_{\mathscr{A}_{\gamma \kappa}} \tilde{c} d A, \text { in the } \gamma \text {-phase. }
\end{aligned}
$$

With the aim of simplifying this expression, the following orders of magnitude estimates are proposed:

$$
\begin{aligned}
& \nabla \cdot\left(\frac{\mathscr{D}}{V_{\gamma}} \int_{\mathscr{A}_{\gamma \kappa}} \mathbf{n} \tilde{c} \quad d A\right)=\mathbf{O}\left(\frac{\mathscr{D} \tilde{c}}{\varepsilon \ell_{\gamma} L}\right), \\
& \mathscr{D} \nabla^{2} \tilde{c}=\mathbf{O}\left(\frac{\mathscr{D} \tilde{c}}{\ell_{\gamma}^{2}}\right), \\
& \mathbf{v} \cdot \nabla \tilde{c}=\mathbf{O}\left(\frac{\tilde{c} v}{\ell_{\gamma}}\right), \\
& \nabla \cdot\langle\tilde{c} \mathbf{v}\rangle^{\gamma}=\mathbf{O}\left(\frac{\tilde{c} v}{L}\right)
\end{aligned}
$$

In these expressions, $v$ denotes the order of magnitude of $\mathbf{v}$ as well as of both $\langle\mathbf{v}\rangle^{\gamma}$ and $\tilde{\mathbf{v}}$ as can be deduced from equation (1d) and the fact that $K n$ is smaller than 0.1 (see also section III A in Lasseux et al., 2014). 
On the basis of the separation of length scales, $\ell_{\gamma} \ll L$, the nonlocal diffusion and convection terms can be assumed to be negligible with respect to their local counterparts. Consequently, equation (9) is reduced to

$$
\frac{\partial \tilde{c}}{\partial t}+\mathbf{v} \cdot \nabla \tilde{c}+\tilde{\mathbf{v}} \cdot \nabla\langle c\rangle^{\gamma}=\mathscr{D} \nabla^{2} \tilde{c}+\frac{k A_{\gamma \kappa}}{V_{\gamma}}\langle c\rangle^{\gamma}+\frac{k}{V_{\gamma}} \int_{\mathscr{A}_{\gamma \kappa}} \tilde{c} \quad d A, \quad \text { in the } \gamma \text {-phase. }
$$

At this point of the developments, it is convenient to examine the characteristic time scale associated to the transport of the concentration deviations, say $\tau$, in order to explore the possibility of a quasi-steady assumption to be applicable. With this in mind, it is worth noting that the average concentration and its gradient are assumed to be constant in both space and time at the closure level. By comparing the orders of magnitude of the terms involving $\tilde{c}$ in equation (11), the following constraint arises that allows assuming quasi-steady conditions:

$$
\mathbf{O}\left(\frac{t_{\mathscr{D}}}{1+P e+\phi^{2} / \varepsilon}\right)=\tau \ll t
$$

Here, $t_{\mathscr{D}} \equiv \ell_{\gamma}^{2} / \mathscr{D}$ is the characteristic time for diffusion at the pore-scale, whereas $P e$ and $\phi^{2}$ are the microscale Péclet number and pore-scale Kinetic number, respectively defined by

$$
\begin{gathered}
P e=v \ell_{\gamma} / \mathscr{D}, \\
\phi^{2}=k \ell_{\gamma} / \mathscr{D},
\end{gathered}
$$

$\phi^{2}$ remaining smaller than 1. It must be noted that the constraint expressed in (12) is usually a severe one and that the macroscale model developed under this constraint remains accurate at times slightly larger than (or even of the order of) $\tau$ (see, for example, results in Figure 7 in Valdés-Parada et al., 2017). Qualitatively, this may be explained by the fact that, even though the effective coefficients might not be accurately predicted from the steady closure problem when this constraint is not satisfied, the relative error induced on the average concentration fields at the corresponding times remains small. Nevertheless, the constraint in (12) is kept here, and, under these circumstances, equation (11) may be reduced to

$$
\mathbf{v} \cdot \nabla \tilde{c}+\underbrace{\tilde{\mathbf{v}} \cdot \nabla\langle c\rangle^{\gamma}}_{\text {source }}=\mathscr{D} \nabla^{2} \tilde{c}+\underbrace{\frac{k A_{\gamma \kappa}}{V_{\gamma}}\langle c\rangle^{\gamma}}_{\text {source }}+\frac{k}{V_{\gamma}} \int_{\mathscr{A}_{\gamma \kappa}} \tilde{c} d A \text {, in the } \gamma \text {-phase. }
$$

The corresponding interfacial boundary condition results from applying the spatial decomposition for the concentration in equation (1e). The result can be expressed as follows:

$$
-\mathbf{n} \cdot(\mathscr{D} \nabla \tilde{\boldsymbol{c}})-\underbrace{\mathbf{n} \cdot\left(\mathscr{D} \nabla\langle c\rangle^{\gamma}\right)}_{\text {source }}=k \tilde{c}+\underbrace{k\langle c\rangle^{\gamma}}_{\text {source }}, \quad \text { at the } \gamma-\kappa \text { interface. }
$$

In addition, a convenient, albeit not mandatory assumption, is that the concentration deviations can be considered as periodic fields in a unit cell. In this way, the following boundary condition is imposed:

$$
\tilde{c}(\mathbf{r})=\tilde{c}\left(\mathbf{r}+\mathbf{l}_{i}\right), \quad i=1,2,3 .
$$

Finally, since the information from the macroscopic boundary conditions is lost, the closure problem is ill-posed, and, for this reason, it is necessary to add the following average constraint for the deviations fields, which results again from the decomposition of the concentration and periodicity:

$$
\langle\tilde{c}\rangle^{\gamma}=0
$$

The problem for $\tilde{c}$ defined by equations (14) being linear, its formal solution can be shown to be given by

$$
\tilde{c}=\mathbf{b} \cdot \nabla\langle c\rangle^{\gamma}+s^{\prime}\langle c\rangle^{\gamma}
$$


where $\mathbf{b}$ and $s^{\prime}$ are closure variables that solve the following problems:

Problem I (source $\left.\nabla\langle c\rangle^{\gamma}\right)$

$$
\begin{gathered}
\tilde{\mathbf{v}}+\mathbf{v} \cdot \nabla \mathbf{b}=\mathscr{D} \nabla^{2} \mathbf{b}+\frac{k}{V_{\gamma}} \int_{\mathscr{A}_{\gamma \kappa}} \mathbf{b} \quad d A, \quad \text { in the } \gamma \text {-phase, } \\
-\mathbf{n} \cdot(\mathscr{D} \nabla \mathbf{b})-k \mathbf{b}=\mathbf{n} \mathscr{D}, \quad \text { at the } \gamma-\kappa \text { interface, } \\
\mathbf{b}\left(\mathbf{r}+\mathbf{l}_{i}\right)=\mathbf{b}(\mathbf{r}), \quad i=1,2,3 \\
\langle\mathbf{b}\rangle^{\gamma}=\mathbf{0} .
\end{gathered}
$$

Problem II (source $\left.\langle c\rangle^{\gamma}\right)$

$$
\begin{gathered}
\mathbf{v} \cdot \nabla s^{\prime}=\mathscr{D} \nabla^{2} s^{\prime}+\frac{k}{V_{\gamma}} \int_{\mathscr{A}_{\gamma \kappa}} s^{\prime} d A+\frac{k A_{\gamma \kappa}}{V_{\gamma}}, \quad \text { in the } \gamma \text {-phase, } \\
-\mathbf{n} \cdot\left(\mathscr{D} \nabla s^{\prime}\right)-k s^{\prime}=k, \quad \text { at the } \gamma-\kappa \text { interface, } \\
s^{\prime}\left(\mathbf{r}+\mathbf{l}_{i}\right)=s^{\prime}(\mathbf{r}), \quad i=1,2,3, \\
\left\langle s^{\prime}\right\rangle^{\gamma}=0 .
\end{gathered}
$$

When nonslip hydrodynamic conditions apply, Problem I, under both reactive and nonreactive conditions, and Problem II have been extensively studied (Edwards et al., 1993; Mauri, 1991; Valdés-Parada et al., 2011). However, the impact of slip flow on the solutions of these problems still needs to be analyzed, and this is the objective of the subsequent sections.

With the formal closure problem solutions available, it is now possible to redirect the attention to the upscaled model and substitute equation (15) into the integral terms in equation (7) in order to obtain the closed form (containing average quantities only) of the macroscopic transport equation for species $A$ that is given by

$$
\begin{aligned}
& \frac{\partial\langle c\rangle^{\gamma}}{\partial t}+\nabla \cdot\left[\left(\langle\mathbf{v}\rangle^{\gamma}+\left\langle\mathbf{v} s^{\prime}\right\rangle^{\gamma}-\frac{\mathscr{D}}{V_{\gamma}} \int_{\mathscr{A}_{\gamma \kappa}} \mathbf{n} s^{\prime} d A+\frac{k}{V_{\gamma}} \int_{\mathscr{A}_{\gamma \kappa}} \mathbf{b} \quad d A\right)\langle c\rangle^{\gamma}\right]= \\
& \nabla \cdot\left\{\left[\mathscr{D}\left(I+\frac{1}{V_{\gamma}} \int_{\mathscr{A}_{\gamma \kappa}} \mathbf{n b} \quad d A\right)-\langle\mathbf{v b}\rangle^{\gamma}\right] \cdot \nabla\langle c\rangle^{\gamma}\right\}-\frac{k A_{\gamma \kappa}}{V_{\gamma}}\left(1+\frac{1}{A_{\gamma \kappa}} \int_{\mathscr{A}_{\gamma \kappa}} s^{\prime} d A\right)\langle c\rangle^{\gamma} .
\end{aligned}
$$

The validity of this result is only subject to the assumptions and constraints adopted at the closure level, namely, the scale hierarchy, the time scale constraint, and periodicity.

In order to express equation (18) in a more compact form, it is of interest to introduce the following effective coefficients:

$$
\begin{gathered}
\mathbf{v}_{e f f}=\langle\mathbf{v}\rangle^{\gamma}+\left\langle\mathbf{v} s^{\prime}\right\rangle^{\gamma}-\frac{\mathscr{D}}{V_{\gamma}} \int_{\mathscr{A}_{\gamma \kappa}} \mathbf{n} s^{\prime} d A+\frac{k}{V_{\gamma}} \int_{\mathscr{A}_{\gamma \kappa}} \mathbf{b} d A, \\
\mathrm{D}^{*}=\mathscr{D}\left(I+\frac{1}{V_{\gamma}} \int_{\mathscr{A}_{\gamma \kappa}} \mathbf{n b} d A\right)-\langle\mathbf{v b}\rangle^{\gamma},
\end{gathered}
$$




$$
k_{e f f}=\frac{k}{A_{\gamma \kappa}} \int_{\mathscr{A}_{\gamma \kappa}}\left(s^{\prime}+1\right) \quad d A,
$$

where $\mathbf{v}_{\text {eff }}$ is the effective convective velocity, $\mathrm{D}^{*}$ the total dispersion tensor, and $k_{\text {eff }}$ the effective reaction rate coefficient. At this point, it is worth noting that the last three terms in equation (19a) account for the influence of reaction on the macroscopic convective mechanism. In addition, the definition of the total dispersion tensor, $D^{*}$, is identical to the one involved under nonreactive transport (see equations (3.3-38)-(3.3-40) in Whitaker, 1999b). However, the dependence of this coefficient on reaction is implicitly contained in the presence of reactive terms in closure Problem I. In addition, all the three effective coefficients depend on slip due to the dependence of the closure variables on $v$. With the above nomenclature, noticing that effective coefficients are constant, equation (18) can be expressed as

$$
\frac{\partial\langle c\rangle^{\gamma}}{\partial t}+\mathbf{v}_{e f f} \cdot \nabla\langle c\rangle^{\gamma}=\mathrm{D}^{*}: \nabla \nabla\langle c\rangle^{\gamma}-k_{e f f} a_{v} \varepsilon^{-1}\langle c\rangle^{\gamma},
$$

where $a_{v}=A_{\gamma \kappa} / V$. This form of the macroscopic transport equation will be referred to as the complete upscaled model. In the following paragraphs, simplifications of this equation are investigated on the basis of orders of magnitude estimates and expansions in the pore-scale Kinetic number, $\phi^{2}$, and Knudsen number, $\mathrm{Kn}$.

\section{Simplified Upscaled Model}

The purpose of this section is to explore the possibility of obtaining a simplified version of the complete upscaled model given in equation (20). This is carried out in two steps, the first one consisting in using order of magnitude analyses and the second one involving the use of Maclaurin expansions.

\subsection{Simplifications Based on Order of Magnitude Estimates}

Simplification is first examined on the different terms that constitute the effective coefficients, more specifically those involved in $\mathbf{v}_{\text {eff }}$ and $k_{\text {eff }}$. To do so, a common aid in the volume averaging approach is to use order of magnitude estimates of the closure variables. This is carried out in Appendix A, and the resulting estimates of these variables can be written as follows:

$$
\begin{gathered}
\mathbf{b}=\mathbf{O}\left(\ell_{\gamma}(1+P e)\right), \quad \text { at the } \gamma-\kappa \text { interface, } \\
s^{\prime}=\mathbf{O}\left(\phi^{2}\right) .
\end{gathered}
$$

It is important to note that both expressions are overestimates, whereas an underestimate for the latter is $s^{\prime}=0$.

With this information at hand, and keeping in mind that $\nabla\langle c\rangle^{\gamma}=\mathbf{O}\left(\langle c\rangle^{\gamma} / L\right)$, the following estimates are proposed for the convective-like terms in equation (18), with the idea that they must be overestimates:

$$
\begin{gathered}
\left(\frac{\mathscr{D}}{V_{\gamma}} \int_{\mathscr{A}_{\gamma \kappa}} \mathbf{n} s^{\prime} \quad d A\right) \cdot \nabla\langle c\rangle^{\gamma}=\mathbf{O}\left(\frac{\mathscr{D} a_{v} \phi^{2}}{\varepsilon L}\langle c\rangle^{\gamma}\right), \\
\left(\frac{k}{V_{\gamma}} \int_{\mathscr{A}_{\gamma \kappa}} \mathbf{b} d A\right) \cdot \nabla\langle c\rangle^{\gamma}=\mathbf{O}\left(\frac{k \ell_{\gamma}(1+P e) a_{v}}{\varepsilon L}\langle c\rangle^{\gamma}\right), \\
\left\langle\mathbf{v} s^{\prime}\right\rangle^{\gamma} \cdot \nabla\langle c\rangle^{\gamma}=\mathbf{O}\left(\frac{v \phi^{2}}{L}\langle c\rangle^{\gamma}\right) .
\end{gathered}
$$

In addition, an order of magnitude estimate of the macroscopic reaction term, that is, the last term on the right-hand side of equation (18), is

$$
k_{e f f} a_{v} \varepsilon^{-1}\langle c\rangle^{\gamma}=\frac{k a_{v}}{\varepsilon} \frac{1}{A_{\gamma \kappa}} \int_{\mathscr{A}_{\gamma \kappa}}\left(s^{\prime}+1\right) \quad d A \quad\langle c\rangle^{\gamma}=\mathbf{O}\left(\frac{k a_{v}}{\varepsilon}\langle c\rangle^{\gamma}\right) .
$$


Here, it is important to note that the order of magnitude estimate for $s^{\prime}$ was taken to be zero with the idea that, in this case, an underestimate of the reaction term is of interest.

On the basis of the length-scale constraint, $\ell_{\gamma} \ll L$, already assumed, a comparison of the estimates given in equations (22a) and (23) leads to conclude that

$$
\left(\frac{\mathscr{D}}{V_{\gamma}} \int_{\mathscr{A}_{\gamma \kappa}} \mathbf{n} s^{\prime} \quad d A\right) \cdot \nabla\langle c\rangle^{\gamma} \ll k_{e f f} a_{v} \varepsilon^{-1}\langle c\rangle^{\gamma} .
$$

In addition, when the constraint

$$
\frac{\ell_{\gamma}}{L}(1+P e) \ll 1
$$

is satisfied, it follows that

$$
\left(\frac{k}{V_{\gamma}} \int_{\mathscr{A}_{\gamma \kappa}} \mathbf{b} \quad d A\right) \cdot \nabla\langle c\rangle^{\gamma} \ll k_{e f f} a_{v} \varepsilon^{-1}\langle c\rangle^{\gamma} .
$$

Finally, upon the constraint

$$
\varepsilon \frac{\ell_{\gamma}}{L} P e \ll 1
$$

it can be inferred that

$$
\left\langle\mathbf{v} s^{\prime}\right\rangle^{\gamma} \cdot \nabla\langle c\rangle^{\gamma} \ll k_{e f f} a_{v} \varepsilon^{-1}\langle c\rangle^{\gamma} .
$$

As mentioned in Appendix A, where (24), (26), and (28) are verified on a numerical example, the simplification resulting from (28) may be valid even if the constraint expressed in (27) is not satisfied. However, in the absence of more precise estimates, it is kept as such in this analysis.

The simplifications on the effective convective velocity in equation (19a) resulting from the above analysis can be summarized as follows:

- Whatever the values of $P e, \phi^{2}$, and $K n$, and on the basis of the constraint $\ell_{\gamma} \ll L$, the expression of $\mathbf{v}_{\text {eff }}$ reduces to

$$
\mathbf{v}_{e f f}=\langle\mathbf{v}\rangle^{\gamma}+\left\langle\mathbf{v} s^{\prime}\right\rangle^{\gamma}+\frac{k}{V_{\gamma}} \int_{\mathscr{A}_{\gamma \kappa}} \mathbf{b} d A .
$$

- In addition, when the constraint

$$
\frac{\ell_{\gamma}}{L} P e \ll 1
$$

is satisfied, $\mathbf{v}_{\text {eff }}=\langle\mathbf{v}\rangle^{\gamma}$. This means that, under these circumstances, the contributions of the reactive correction to convective transport can be ignored in the macroscopic model given in equation (18).

- Finally, from the expression of $k_{\text {eff }}$ and $\mathbf{v}_{\text {eff }}$ in equations (19c) and (29), respectively, together with the order of magnitude estimate for $s^{\prime}$ in equation (21b), it can be inferred that, when $\phi^{2} \ll 1$,

$$
k_{e f f}=k
$$

and

$$
\mathbf{v}_{e f f}=\langle\mathbf{v}\rangle^{\gamma}+\frac{k}{V_{\gamma}} \int_{\mathscr{A}_{\gamma \kappa}} \mathbf{b} \quad d A .
$$

In the following paragraphs, simplifications of the total dispersion tensor $\mathrm{D}^{*}$ are sought by making use of Maclaurin expansions. 


\subsection{Maclaurin Expansions}

With the intention of further simplifying the macroscopic model, it is convenient to consider Maclaurin expansions in terms of the Kinetic number (see also Mauri, 1991 for a similar approach for no-slip conditions). To do this, the following change of variable may be first used:

$$
s=s^{\prime}+1
$$

which yields a simpler form of Problem II given by

$$
\begin{gathered}
\mathbf{v} \cdot \nabla s=\mathscr{D} \nabla^{2} s+\frac{k}{V_{\gamma}} \int_{\mathscr{A}_{\gamma \kappa}} s \quad d A, \quad \text { in the } \gamma \text {-phase, } \\
-\mathbf{n} \cdot(\mathscr{D} \nabla s)=k s, \quad \text { at the } \gamma-\kappa \text { interface, } \\
s\left(\mathbf{r}+\mathbf{l}_{i}\right)=s(\mathbf{r}), \quad i=1,2,3, \\
\langle s\rangle^{\gamma}=1 .
\end{gathered}
$$

It should be noted that $s=\mathbf{O}(1)$. In this way, the definition of $k_{\text {eff }}$ given by equation (19c) can be rewritten as

$$
k_{e f f}=\frac{k}{A_{\gamma \kappa}} \int_{\mathscr{A}_{\gamma \kappa}} s \quad d A .
$$

In addition, $s^{\prime}$ can be replaced by $s$ in the expression of $\mathbf{v}_{\text {eff }}$ in equation (29) to obtain

$$
\mathbf{v}_{e f f}=\langle\mathbf{v} s\rangle^{\gamma}+\frac{k}{V_{\gamma}} \int_{\mathscr{A}_{\gamma \kappa}} \mathbf{b} d A .
$$

At this stage, a rescaling making variables of order 1 is used. It is given by

$$
\mathbf{b}^{*}=\frac{\mathbf{b}}{\ell_{\gamma}(1+P e)} ; \quad \nabla^{*}=\ell_{\gamma} \nabla ; \quad \mathbf{v}^{*}=\frac{\mathbf{v}}{v} .
$$

The dimensionless forms of the closure problems can hence be expressed as

Problem I*

$$
\begin{gathered}
\frac{P e}{1+P e} \tilde{\mathbf{v}}^{*}+P e \mathbf{v}^{*} \cdot \nabla^{*} \mathbf{b}^{*}=\nabla^{* 2} \mathbf{b}^{*}+\frac{\phi^{2}}{V_{\gamma}^{*}} \int_{\mathscr{A}_{\gamma \kappa}} \mathbf{b}^{*} d A^{*}, \quad \text { in the } \gamma \text {-phase, } \\
-\mathbf{n} \cdot \nabla^{*} \mathbf{b}^{*}-\phi^{2} \mathbf{b}^{*}=\frac{1}{1+P e} \mathbf{n}, \quad \text { at the } \gamma-\kappa \text { interface, } \\
\mathbf{b}^{*}\left(\mathbf{r}+\mathbf{l}_{i}\right)=\mathbf{b}^{*}(\mathbf{r}), \quad i=1,2,3, \\
\left\langle\mathbf{b}^{*}\right\rangle^{\gamma}=\mathbf{0} .
\end{gathered}
$$

Problem II

$$
\begin{gathered}
P e \mathbf{v}^{*} \cdot \nabla^{*} s=\nabla^{* 2} s+\frac{\phi^{2}}{V_{\gamma}^{*}} \int_{\mathscr{A}_{\gamma \kappa}} s \quad d A^{*}, \quad \text { in the } \gamma \text {-phase, } \\
-\mathbf{n} \cdot \nabla^{*} s=\phi^{2} s, \quad \text { at the } \gamma-\kappa \text { interface, } \\
s\left(\mathbf{r}+\mathbf{l}_{i}\right)=s(\mathbf{r}), \quad i=1,2,3, \\
\langle s\rangle^{\gamma}=1 .
\end{gathered}
$$


Accordingly, the dimensionless version of the total dispersion tensor, effective velocity, and effective reaction rate coefficient can be reformulated as

$$
\begin{gathered}
\frac{\mathrm{D}^{*}}{\mathscr{D}}=I+(1+P e)\left(\frac{1}{V_{\gamma}^{*}} \int_{\mathscr{A}_{\gamma \kappa}} \mathbf{n} \mathbf{b}^{*} d A^{*}-P e\left\langle\mathbf{v}^{*} \mathbf{b}^{*}\right\rangle^{\gamma}\right), \\
\frac{\mathbf{v}_{e f f}}{v}=\left\langle\mathbf{v}^{*} s\right\rangle^{\gamma}+\phi^{2} \frac{1+P e}{P e} \frac{1}{V_{\gamma}^{*}} \int_{\mathscr{A}_{\gamma \kappa}} \mathbf{b}^{*} d A^{*}, \\
\frac{k_{e f f}}{k}=\frac{1}{A_{\gamma \kappa}^{*}} \int_{\mathscr{A}_{\gamma \kappa}} s d A^{*} .
\end{gathered}
$$

At this point, it must be noted that when $\phi^{2} \ll 1$, the reaction terms can be neglected in Problem $I^{*}$ and Problem II*. Hence, Problem $I^{*}$ is the same as for passive dispersion (see equations (3.3-35) in Whitaker, 1999b), and so is D*, whereas, for Problem $I^{*}, s=1$. In that case, no other simplification than those already expressed in equation (31) can be obtained. For this reason, $\phi^{2}=\mathbf{O}(1)$ is considered in the remainder of this section. In addition, only a simplification on $D^{*}$ is expected from Maclaurin expansions. Consequently, these expansions only need to be carried out on Problem I*.

The orders of magnitude of the terms involving Pe in closure Problem $I^{*}$ differ depending on the transport regime defined by the Péclet number. This requires special attention in the process of expanding the closure variable $\mathbf{b}^{*}$ in terms of the Kinetic number and in the identification of the ensuing subproblems at the successive orders in $\phi^{2}$. When transport is dominated by diffusion (i.e., $P e \ll 1$ ), the dispersion tensor reduces to the effective diffusivity tensor. This situation was explored by Valdés-Parada et al. (2017) where it was shown that this effective coefficient can be considered as reaction-independent. For this reason, situations for which $P e \geqslant 1$ will be considered in the following paragraphs.

\subsubsection{Expansions in Terms of the Kinetic Number- $P e=O(1)$}

When convection and diffusion are of equal importance in the transport process (i.e., $P e=\mathbf{O}(1)$ ), all the terms in closure Problem $I^{*}$ in equations (37) are $\mathbf{O}(1)$. In this case, assuming $\phi^{2}<1$, the following expansion of the closure variable $\mathbf{b}^{*}$ in terms of the Kinetic number is considered:

$$
\mathbf{b}^{*}=\sum_{j=0}^{\infty}\left(\phi^{2}\right)^{j} \mathbf{b}_{j}^{*}
$$

When this expansion is substituted into equations (37), closure Problem I* yields the following closure problems at the 0 th and $j$ th $(j \geqslant 1)$ orders, respectively given by

Problem $I_{0}^{*}$

$$
\begin{gathered}
\frac{P e}{1+P e} \tilde{\mathbf{v}}^{*}+P e \mathbf{v}^{*} \cdot \nabla^{*} \mathbf{b}_{0}^{*}=\nabla^{* 2} \mathbf{b}_{0}^{*}, \quad \text { in the } \gamma \text {-phase, } \\
-\mathbf{n} \cdot \nabla^{*} \mathbf{b}_{0}^{*}=\frac{1}{1+P e} \mathbf{n}, \quad \text { at the } \gamma-\kappa \text { interface, } \\
\mathbf{b}_{0}^{*}\left(\mathbf{r}+\mathbf{l}_{i}\right)=\mathbf{b}_{0}^{*}(\mathbf{r}), \quad i=1,2,3 \\
\left\langle\mathbf{b}_{0}^{*}\right\rangle^{\gamma}=\mathbf{0}
\end{gathered}
$$

Problem $I_{j}^{*}(j \geqslant 1)$

$$
P e \mathbf{v}^{*} \cdot \nabla^{*} \mathbf{b}_{j}^{*}=\nabla^{* 2} \mathbf{b}_{j}^{*}+\frac{1}{V_{\gamma}^{*}} \int_{\mathscr{A}_{\gamma \kappa}} \mathbf{b}_{j-1}^{*} d A^{*}, \quad \text { in the } \gamma \text {-phase, }
$$


$-\mathbf{n} \cdot \nabla^{*} \mathbf{b}_{j}^{*}=\mathbf{b}_{j-1}^{*}, \quad$ at the $\gamma-\kappa$ interface,

$$
\mathbf{b}_{j}^{*}\left(\mathbf{r}+\mathbf{l}_{i}\right)=\mathbf{b}_{j}^{*}(\mathbf{r}), \quad i=1,2,3,
$$

$$
\left\langle\mathbf{b}_{j}^{*}\right\rangle^{\gamma}=\mathbf{0}
$$

Note that Problem $I_{0}^{*}$ corresponds to the typical closure problem encountered in passive dispersion in porous media (see equations (3.3-35) in Whitaker, 1999b).

As expected, the order of magnitude analysis of the above closure problems indicates that, at most,

$$
\mathbf{b}_{j}^{*}=\mathbf{O}(1), \quad j \geqslant 0,
$$

in the bulk of the $\gamma$-phase and at the $\gamma-\kappa$ interface.

When the expansion in equation (40) is substituted in the definition of $D^{*}$, the following expression results:

$$
\begin{aligned}
& \frac{\mathrm{D}^{*}}{\mathscr{D}}=\underbrace{\mathrm{I}+(1+P e)\left(\frac{1}{V_{\gamma}^{*}} \int_{\mathscr{A}_{\gamma \kappa}} \mathbf{n} \mathbf{b}_{0}^{*} d A^{*}-P e\left\langle\mathbf{v}^{*} \mathbf{b}_{0}^{*}\right\rangle^{\gamma}\right)}_{\mathrm{D}_{0}^{*} / \mathscr{D}} \\
& +(1+P e) \sum_{j=1}^{\infty}\left(\phi^{2}\right)^{j} \underbrace{\left(\frac{1}{V_{\gamma}^{*}} \int_{\mathscr{A}_{\gamma \kappa}} \mathbf{n b}_{j}^{*} d A^{*}-P e\left\langle\mathbf{v}^{*} \mathbf{b}_{j}^{*}\right\rangle^{\gamma}\right)}_{\mathrm{D}_{j}^{*} / \mathscr{D}}
\end{aligned}
$$

From this expression, the following order of magnitude estimates for the $j$ th $(j \geqslant 1)$ dispersive terms emerging from the series in the expression of $D^{*} / \mathscr{D}$ in equation (44) are proposed:

$$
\begin{gathered}
\left(\frac{\mathscr{D}(1+P e)\left(\phi^{2}\right)^{j}}{V_{\gamma}^{*}} \int_{\mathscr{A}_{\gamma \kappa}} \mathbf{n b}_{j}^{*} \quad d A^{*}\right): \nabla \nabla\langle c\rangle^{\gamma}=\mathbf{O}\left(\frac{k\langle c\rangle^{\gamma}}{\varepsilon \ell_{\gamma}}\left(\frac{\ell_{\gamma}}{L}\right)^{2} \phi^{2(j-1)}\right), \\
\left(\mathscr{D}\left(\phi^{2}\right)^{j} P e(1+P e)\left\langle\mathbf{v}^{*} \mathbf{b}_{j}^{*}\right\rangle^{\gamma}\right): \nabla \nabla\langle c\rangle^{\gamma}=\mathbf{O}\left(\frac{\nu\langle c\rangle^{\gamma}}{L} \frac{\ell_{\gamma}}{L} \phi^{2 j}\right) .
\end{gathered}
$$

Note that the series are convergent because the successive terms decrease as $j$ increases. The estimate in equation (45a) can be compared with the one given in equation (23) while the estimate in equation (45b) is compared to the convective term for which the order of magnitude is

$$
\langle\mathbf{v}\rangle^{\gamma} \cdot \nabla\langle c\rangle^{\gamma}=\mathbf{O}\left(\frac{\langle c\rangle^{\gamma} v}{L}\right)
$$

Therefore, since the present analysis is restricted to $\phi^{2}<1$, and on the basis of the length-scale constraint $\ell_{\gamma} \ll L$, it can be deduced that the following comparisons are reasonable:

$$
\begin{gathered}
\left(\sum_{j=1}^{\infty} \frac{\mathscr{D}(1+P e)\left(\phi^{2}\right)^{j}}{V_{\gamma}^{*}} \int_{\mathscr{A}_{\gamma \kappa}} \mathbf{n b}_{j}^{*} \quad d A^{*}\right): \nabla \nabla\langle c\rangle^{\gamma} \ll k_{e f f} a_{v} \varepsilon^{-1}\langle c\rangle^{\gamma}, \\
\left(\mathscr{D}(1+P e) \sum_{j=1}^{\infty}\left(\phi^{2}\right)^{j} P e\left\langle\mathbf{v}^{*} \mathbf{b}_{j}^{*}\right\rangle^{\gamma}\right): \nabla \nabla\langle c\rangle^{\gamma} \ll\langle\mathbf{v}\rangle^{\gamma} \cdot \nabla\langle c\rangle^{\gamma} .
\end{gathered}
$$


Consequently, the macroscale model can be written in the following simplified form:

$$
\frac{\partial\langle c\rangle^{\gamma}}{\partial t}+\langle\mathbf{v}\rangle^{\gamma} \cdot \nabla\langle c\rangle^{\gamma}=\mathrm{D}_{0}^{*}: \nabla \nabla\langle c\rangle^{\gamma}-k_{e f f} a_{v} \varepsilon^{-1}\langle c\rangle^{\gamma}
$$

with

$$
\frac{\mathrm{D}_{0}^{*}}{\mathscr{D}}=\mathrm{I}+(1+P e)\left(\frac{1}{V_{\gamma}^{*}} \int_{\mathscr{A}_{\gamma \kappa}} \mathbf{n} \mathbf{b}_{0}^{*} \quad d A^{*}-P e\left\langle\mathbf{v}^{*} \mathbf{b}_{0}^{*}\right\rangle^{\gamma}\right)
$$

This effective coefficient corresponds to the total dispersion tensor under nonreactive conditions. In the following section, the analysis is extended to situations for which $P e \gg 1$.

5.2.2. Expansions in Terms of the Kinetic and Péclet Numbers - Pe $\gg 1$

When $P e \gg 1$, the convective terms in equation (37a) are much larger than 1, and this represents a difficulty in the identification of the problems at the successive orders of $\phi^{2}$ if the expansion given by equation (40) were employed. For this reason, $\mathbf{b}^{*}$ is first expanded in terms of $P e^{-1}$ as

$$
\mathbf{b}^{*}=\sum_{j=0}^{\infty} P e^{-j} \mathbf{b}_{j}^{*}
$$

This expansion is then introduced in equations (37) after equation (37a) is divided by $P e$. The subproblems at the successive orders of $P e^{-1}$ are then identified, taking into account the fact that $\phi^{2}=\mathbf{O}(1)$. At this stage, a second expansion in $\phi^{2}$ defined as

$$
\mathbf{b}_{j}^{*}=\sum_{k=0}^{\infty}\left(\phi^{2}\right)^{k} \mathbf{b}_{j k}^{*}
$$

is employed in each subproblem at the $j$ th order of $P e^{-1}$. Problems at the successive $j$ th and $k$ th orders of $P e^{-1}$ and $\phi^{2}$ are then identified. Although they are not provided here for the sake of brevity, $\mathbf{b}_{j k}^{*}$ is found to be $\mathbf{O}(1)$, as expected. Accordingly, this double expansion of $\mathbf{b}^{*}$ can be inserted in the expression of $D^{*}$. Using the notation

$$
\mathbf{b}_{k}^{+}=\sum_{j=0}^{\infty} P e^{-j} \mathbf{b}_{j k}^{*}
$$

yields

$$
\begin{aligned}
\frac{\mathrm{D}^{*}}{\mathscr{D}}=\mathrm{I} & +(1+P e)\left(\frac{1}{V_{\gamma}^{*}} \int_{\mathscr{A}_{\gamma \kappa}} \mathbf{n} \mathbf{b}_{0}^{+} d A^{*}-P e\left\langle\mathbf{v}^{*} \mathbf{b}_{0}^{+}\right\rangle^{\gamma}\right) \\
& +(1+P e) \sum_{k=1}^{\infty}\left(\phi^{2}\right)^{k}\left(\frac{1}{V_{\gamma}^{*}} \int_{\mathscr{A}_{\gamma \kappa}} \mathbf{n b}_{k}^{+} d A^{*}-P e\left\langle\mathbf{v}^{*} \mathbf{b}_{k}^{+}\right\rangle^{\gamma}\right) .
\end{aligned}
$$

The closure problem for $\mathbf{b}_{k}^{+}(k \geqslant 0)$ can then be derived. It is obtained by adding the problems on $\mathbf{b}_{j k}^{*}$ at the appropriate $j$ th orders after multiplication by the convenient power of $P e$ for each of them. This results in closure problems that are exactly identical to problems $I_{0}^{*}$ and $I_{j}^{*}(j \geqslant 1)$ in equations 41 and 42 in which $\mathbf{b}_{j}^{*}$ is formally replaced by $\mathbf{b}_{j}^{+}(j \geqslant 0)$. Consequently, $\mathbf{b}_{j}^{+}=\mathbf{b}_{j}^{*}(j \geqslant 0)$, which are all $\mathbf{O}(1)$.

The simplification on $D^{*}$ can now be analyzed on the basis of the following order of magnitude estimates:

$$
\begin{gathered}
\left(\frac{\mathscr{D}(1+P e)\left(\phi^{2}\right)^{k}}{V_{\gamma}^{*}} \int_{\mathscr{A}_{\gamma \kappa}} \mathbf{n b}_{k}^{+} \quad d A^{*}\right): \nabla \nabla\langle c\rangle^{\gamma}=\mathbf{O}\left(\frac{k(1+P e)\langle c\rangle^{\gamma}}{\varepsilon \ell_{\gamma}}\left(\frac{\ell_{\gamma}}{L}\right)^{2} \phi^{2(k-1)}\right), \\
\left(\mathscr{D}\left(\phi^{2}\right)^{k} P e(1+P e)\left\langle\mathbf{v}^{*} \mathbf{b}_{k}^{+}\right\rangle^{\gamma}\right): \nabla \nabla\langle c\rangle^{\gamma}=\mathbf{O}\left(\frac{v\langle c\rangle^{\gamma}}{L}(1+P e) \frac{\ell_{\gamma}}{L} \phi^{2 k}\right) .
\end{gathered}
$$


These estimates can be compared with those given in equations (23) and (46), respectively. Since $\phi^{2}<1$, the length-scale constraints

$$
\begin{gathered}
(1+P e)\left(\frac{\ell_{\gamma}}{L}\right)^{2} \ll 1, \\
\phi^{2}(1+P e) \frac{\ell_{\gamma}}{L} \ll 1,
\end{gathered}
$$

suggest that the following comparisons are reasonable:

$$
\begin{gathered}
\left(\sum_{k=1}^{\infty} \frac{\mathscr{D}(1+P e)\left(\phi^{2}\right)^{k}}{V_{\gamma}^{*}} \int_{\mathscr{A}_{\gamma \kappa}} \mathbf{n b}_{k}^{+} \quad d A^{*}\right): \nabla \nabla\langle c\rangle^{\gamma} \ll k_{e f f} a_{v} \varepsilon^{-1}\langle c\rangle^{\gamma}, \\
\left(\mathscr{D} \sum_{k=1}^{\infty}\left(\phi^{2}\right)^{k} P e(1+P e)\left\langle\mathbf{v}^{*} \mathbf{b}_{k}^{+}\right\rangle^{\gamma}\right): \nabla \nabla\langle c\rangle^{\gamma} \ll\langle\mathbf{v}\rangle^{\gamma} \cdot \nabla\langle c\rangle^{\gamma} .
\end{gathered}
$$

At this point, it is pertinent to note that the constraints given in 55 can be summarized into $P e \ell_{\gamma} / L \ll 1$, which was already imposed to simplify $\mathbf{v}_{\text {eff }}$. Consequently, in the convection-dominated regime, and under the latter constraint, the macroscale model can be written in the simplified form given in equation (48).

The above simplifications show that the macroscopic dispersive and convective mechanisms can be decoupled from the heterogeneous reaction whenever $\mathrm{Pe} \ell_{\gamma} / L \ll 1$ so that $\mathbf{v}_{\text {eff }} \approx\langle\mathbf{v}\rangle^{\gamma}$ and the total dispersion coefficient can be reasonably estimated from the closure problem at the 0 th order of $\phi^{2}$ given by equations 41 , which is identical to the one in the absence of reaction (Whitaker, 1999b). This is in agreement with the conclusion reached in the work by Mauri (1991) and generalizes the result in the presence or absence of hydrodynamic slip.

\subsubsection{Final Forms of the Upscaled Models and Closure Problems}

In the recent work by Lasseux et al. (2016), it was recalled that the apparent permeability tensor, $\mathrm{K}_{s}$, in equation (8) does not reduce, in general, to the intrinsic permeability tensor when the Knudsen number is not vanishingly small. Moreover, it was suggested that a finite truncation of an expansion of $\mathrm{K}_{s}$ in terms of the Knudsen number was suitable to provide an accurate prediction of the apparent permeability tensor. This motivates applying a similar approach to the effective coefficients involved in the present analysis. However, when this is done, it is found that all the terms in the expansion in the Knudsen number are required. As a consequence, no further simplifications with respect to this parameter are possible.

To conclude this section, the upscaled models with the associated constraints and the related closure problems can be summarized as follows.

- Under the constraints on the physical parameters expressed by $R e \ll 1, M a^{2} \ll 1, M a \ll F r, K n \lesssim 0.1$ and on the time-scale and length-scale constraints given by $t \gg \max \left(\operatorname{Re} \ell_{\gamma} / v,\left(\ell_{\gamma}^{2} / \mathscr{D}\right) /\left(1+v \ell_{\gamma} / \mathscr{D}+\phi^{2} / \varepsilon\right)\right)$ and $\ell_{\gamma} \ll L$, further assuming that the total concentration of all species remains constant, the macroscopic mass conservation equation governing dispersion and heterogeneous reaction of a dilute species in the presence of hydrodynamic slip is given by

$$
\frac{\partial\langle c\rangle^{\gamma}}{\partial t}+\mathbf{v}_{e f f} \cdot \nabla\langle c\rangle^{\gamma}=\mathrm{D}^{*}: \nabla \nabla\langle c\rangle^{\gamma}-k_{e f f} a_{v} \varepsilon^{-1}\langle c\rangle^{\gamma}
$$

In this model, $\mathbf{v}_{\text {eff }}, \mathrm{D}^{*}$, and $k_{\text {eff }}$ are given by equations 39 , which are determined by the solutions of the closure problems $I^{*}$ and $I I^{*}$ in equations 37 and 38. No constraint on $P e$ or $\phi^{2}$ is associated to this model that is referred to as the complete upscaled model.

- When $\phi^{2} \ll 1$, the three effective coefficients are simplified to the following forms:

$$
\frac{D^{*}}{\mathscr{D}}=\frac{\mathrm{D}_{0}^{*}}{\mathscr{D}}=\mathrm{I}+(1+P e)\left(\frac{1}{V_{\gamma}^{*}} \int_{\mathscr{A}_{\gamma \kappa}} \mathbf{n b}_{0}^{*} \quad d A^{*}-P e\left\langle\mathbf{v}^{*} \mathbf{b}_{0}^{*}\right\rangle^{\gamma}\right),
$$




$$
\begin{gathered}
\frac{\mathbf{v}_{e f f}}{v}=\left\langle\mathbf{v}^{*}\right\rangle^{\gamma}+\phi^{2} \frac{1+P e}{P e} \frac{1}{V_{\gamma}^{*}} \int_{\mathscr{A}_{\gamma \kappa}} \mathbf{b}_{0}^{*} d A^{*}, \\
\frac{k_{e f f}}{k}=1,
\end{gathered}
$$

where $\mathbf{b}_{0}^{*}$ is solution of closure Problem $I_{0}^{*}$ as given in equations 41 . The symmetry properties of the tensor $\mathrm{D}_{0}^{*}$ were carefully explored by Lasseux and Valdés-Parada (2017).

- When $\phi^{2}<1$ and the constraint $P e \ell_{\gamma} / L \ll 1$ is satisfied, the effective coefficients are such that $\mathrm{D}^{*} / \mathscr{D}=$ $\mathrm{D}_{0}^{*} / \mathscr{D}$ (see equation (58a)), $k_{\text {eff }} / k$ is given by equations (39c), whereas

$$
\frac{\mathbf{v}_{e f f}}{v}=\left\langle\mathbf{v}^{*}\right\rangle^{\gamma}
$$

In this last case, the macroscopic equation is referred to as the simplified upscaled model.

It must be recalled that the constraint $\mathrm{Pe} \ell_{\gamma} / L \ll 1$ is the result of an analysis based upon order of magnitude estimates, and it is thus likely that the simplified model has a range of applicability beyond the one strictly limited by this constraint. For this reason, it is desirable to carry out numerical evaluations of the effective coefficients and of the average concentration resulting from both the simplified and complete upscaled models.

Before proceeding with the numerical simulations, it is worth noting that the last term on the right-hand side of equation (38a) can be reformulated as

$$
\frac{\phi^{2}}{V_{\gamma}^{*}} \int_{\mathscr{A}_{\gamma \kappa}} s \quad d A^{*}=\frac{A_{\gamma \kappa}^{*} k_{e f f} \phi^{2}}{k V^{*} \varepsilon}=\frac{k_{e f f}}{k} \phi^{2} a_{\nu}^{*} \varepsilon^{-1} .
$$

This suggests proposing the final change of variable

$$
s^{*}=\frac{s}{\frac{\phi^{2}}{V_{\gamma}^{*}} \int_{\mathscr{A}_{\gamma \kappa}} S d A^{*}}
$$

so that Problem II* can be written as

Problem II*

$$
\begin{gathered}
P e \mathbf{v}^{*} \cdot \nabla^{*} s^{*}=\nabla^{* 2} s^{*}+1, \quad \text { in the } \gamma \text {-phase, } \\
-\mathbf{n} \cdot \nabla^{*} s^{*}=\phi^{2} s^{*}, \quad \text { at the } \gamma-\kappa \text { interface, } \\
s^{*}\left(\mathbf{r}+\mathbf{l}_{i}\right)=s^{*}(\mathbf{r}), \quad i=1,2,3,
\end{gathered}
$$

which represents an easier form for a numerical solution. From this problem, one may then compute the effective reaction rate coefficient as

$$
\frac{k_{e f f}}{k}=\frac{\varepsilon}{\left\langle s^{*}\right\rangle^{\gamma} \phi^{2} a_{v}^{*}} .
$$

\section{Results}

The purpose of this section is twofold. Firstly, the dependence of the effective coefficients is explored in terms of the dimensionless numbers $K n, P e, \phi^{2}$, and porosity. Secondly, the upscaled models are validated by comparison with predictions of the dynamics of the average concentration resulting from direct numerical simulations. 


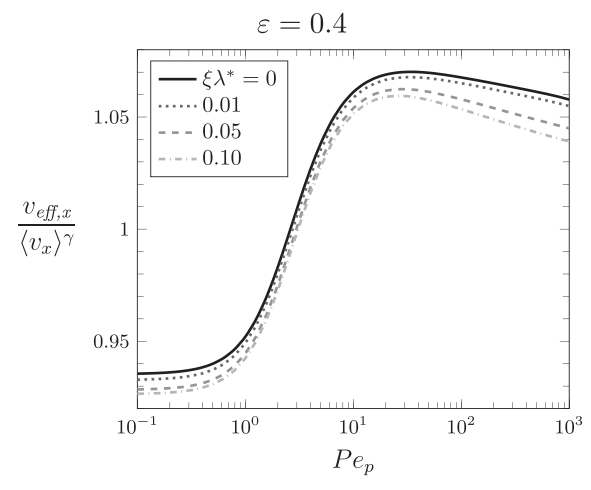

a)

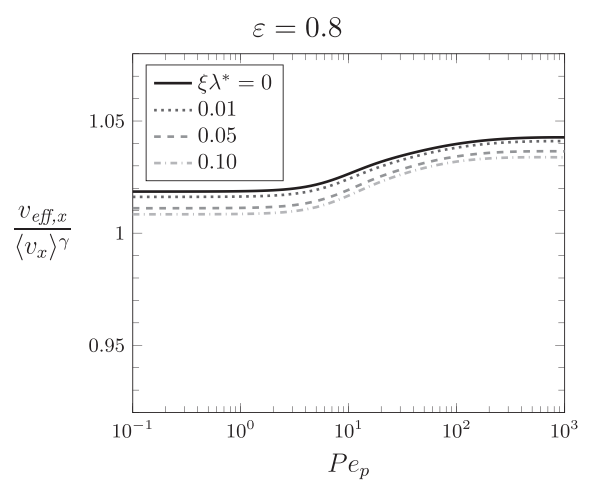

b)

Figure 3. Examples of the predictions of the $x$-component of the effective velocity in the complete upscaled model normalized with the intrinsic average of the $x$-component of the fluid velocity as a function of the particle Péclet number for $\varepsilon=0.4$ (a) and $\varepsilon=0.8(\mathrm{~b})$, taking $\phi_{c}^{2}=1$.

\subsection{Predictions of the Effective Medium Coefficients}

In order to analyze the effective-medium coefficients involved in the complete and simplified versions of the upscaled model, the following strategy is used: 1) The dimensionless versions of the pore-scale equations for total mass and momentum transport are solved in a periodic unit cell to obtain $\mathbf{v}^{*}$. 2) The result is used in the solution of closure problems $I^{*}$ (equations 37), $I_{0}^{*}$ (equations 41), and $I I^{*}$ (equations 62). 3) Solutions of these closure problems are employed to compute the effective medium coefficients involved in both the complete and simplified upscaled models. Furthermore, since the closure problems only depend on the Knudsen number by means of the fluid velocity, only steps 2 and 3 need to be repeated when changing the Péclet number or the Kinetic number values. The numerical solutions of the closure problems were obtained using the finite element solver Comsol 5.4 in the simple periodic unit cell sketched in Figure 2, taking porosity values of 0.4 and 0.8 . Certainly, more complex unit cells could be considered. For example, in the work by Valdés-Parada et al. (2011), it is shown that the predicted values of the longitudinal and transverse components of the total dispersion tensor resulting from three-dimensional unit cells are larger than those obtain with two-dimensional unit cells for conditions in which $P e \ll 1$ while the opposite holds for $P e \gg 1$. These authors considered a cubic block and a square to represent the solid phase in the threeand two-dimensional periodic unit cells, respectively, keeping the same porosity value. In the more recent work by Yan and Li (2017), predictions obtained on random distributions of solid obstacles in the unit cell were reported. In the present work, the simple periodic unit cell shown in Figure 2 is chosen for illustrative purposes.

It is worth mentioning that conventional meshing analyses were performed in order to guarantee the independence of the predictions with this numerical parameter. In the following, results are presented in terms of the particle Péclet number, which is defined as

$$
P e_{p}=\frac{\left\|\langle\mathbf{v}\rangle^{\gamma}\right\| \ell_{\kappa}}{\mathscr{D}} \frac{\varepsilon}{1-\varepsilon}=\frac{v \ell_{\kappa}}{\mathscr{D}} \frac{\varepsilon}{1-\varepsilon} .
$$

In order to avoid numerical difficulties, the simulations were carried out for $P e_{p}$ values smaller than $10^{3}$, which correspond to values of $P e=\mathbf{O}(100)$. In addition, the slip flow effects are analyzed with respect to the cell Knudsen number, $\lambda^{*}$, defined as $\lambda^{*}=K n \ell_{\gamma} / \ell=\lambda / \ell$, and the reaction effects are studied in terms of the cell Kinetic number $\phi_{c}^{2}=\phi^{2} \ell / \ell_{\gamma}$.

To commence the analysis, let attention be directed towards the predictions of $\mathbf{v}_{\text {eff }}$ in the complete upscaled model. Due to the symmetric geometry of the unit cell, it is sufficient to focus on the $x$-component, $v_{e f f, x}$. In Figure 3, the dependence of $v_{\text {eff } x}$ upon $P e_{p}$ is presented, normalized with $\left\langle v_{x}\right\rangle^{\gamma}$, taking $\phi_{c}^{2}=1$, the two porosity values $\varepsilon=0.4$ and $\varepsilon=0.8$, and four values of $\xi \lambda^{*}$. The results in Figures $3 \mathrm{a}$ and $3 \mathrm{~b}$ suggest that the effect of $\phi_{c}^{2}$ on $v_{e f f, x}$ is moderate, since there is less than $10 \%$ of difference with respect to $\left\langle v_{x}\right\rangle^{\gamma}$, for $\varepsilon=0.4$. As the porosity increases, this effect is reduced because the interfacial area also decreases in the case under study here. Furthermore, slip effects are mildly influential on the approximation of $v_{\text {eff, } x}$ by $\left\langle v_{x}\right\rangle$. These results are consistent with those reported in section A2 of Appendix A. Indeed, they show that the approximation 


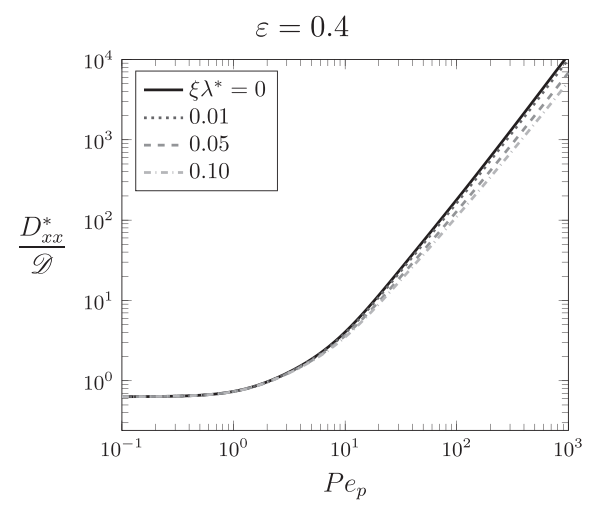

a)

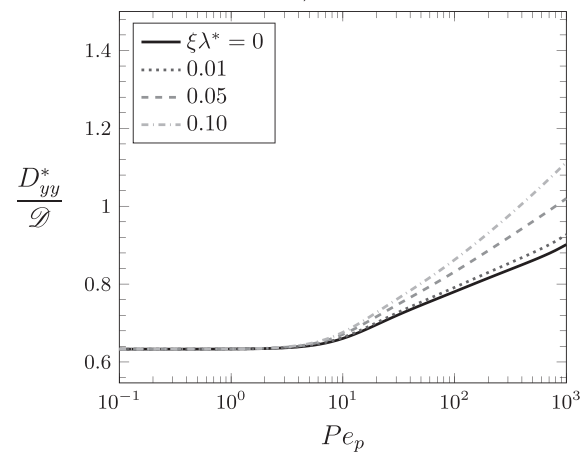

c)

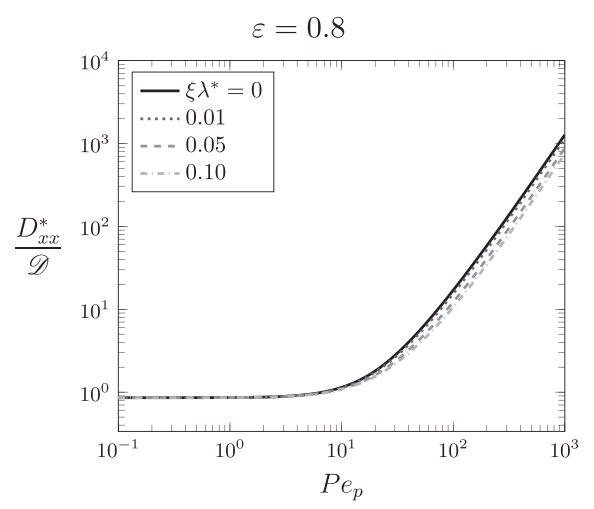

b)

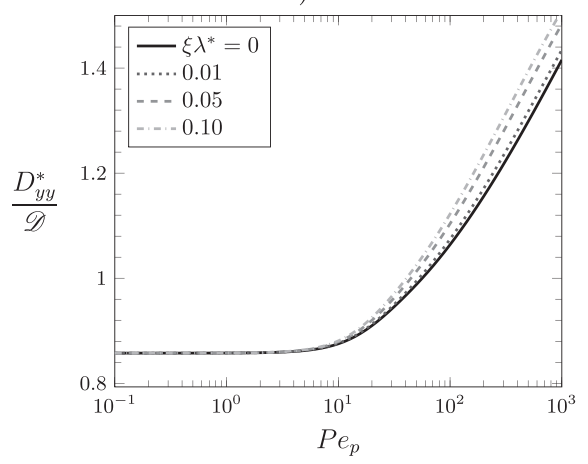

d)

Figure 4. Longitudinal ( $a$ and $b$ ) and transverse ( $c$ and d) components of the dispersion tensor $D^{*}$ versus the particle Péclet number $P e_{p}$ for $\phi_{c}^{2}=1.0$ and four values of the cell Knudsen number $\lambda^{*}$. The porosity values are $\varepsilon=0.4$ (a and c) and $\varepsilon=0.8$ (b and d).

$v_{e f f, x}=\left\langle v_{x}\right\rangle^{\gamma}$ seems valid for any Péclet number value, thus suggesting that the constraint $P e \ell_{\gamma} / L \ll 1$ may be overly severe at least for this effective medium coefficient.

Directing the attention to the total dispersion tensor in the complete upscaled model, the predictions on the longitudinal and transverse coefficients, $D_{x x}^{*} / \mathscr{D}$ and $D_{y y}^{*} / \mathscr{D}$, are first represented versus $P e_{p}$ in Figure 4 for four values of $\xi \lambda^{*}, \phi_{c}^{2}=1, \varepsilon=0.4$, and $\varepsilon=0.8$. Second, the same results, but normalized with the corresponding components of $\mathrm{D}_{0}^{*}$ (i.e., $\mathrm{D}^{*}$ when $\phi_{c}^{2}=0$ ), which is also involved in the simplified model, are considered. They are reported versus $P e_{p}$ in Figure 5 for the same values of the porosity, $\xi \lambda^{*}$ and $\phi_{c}^{2}$.

Results in Figure 4 show that the longitudinal dispersion decreases with $\xi \lambda^{*}$ while the opposite holds for the transverse component. This tendency can be inferred from the smoothing properties of the hydrodynamic slip on the velocity profiles, thus decreasing the values of the velocity spatial deviations in the direction of the mean flow while the opposite is true in the transverse direction. This has a direct impact on the components of the total dispersion tensor as can be seen from the expression in equation (19b). In fact, for the longitudinal dispersion, the values corresponding to $P e_{p}=1,000$ and $\xi \lambda^{*}=0.1$ are approximately half of those corresponding to $\xi \lambda^{*}=0$, and this trend is likely to increase with the Péclet number.

As in the analysis of $v_{\text {eff. } x}$, results in Figure 5 show that the effect of reaction on the components of the dispersion coefficients is more pronounced for $\varepsilon=0.4$. In that case, the relative difference can surpass $10 \%$, in particular for the transverse dispersion coefficient. Moreover, as slip effects are increased, the influence of reaction is damped, which is consistent with the observations made in Figure 3 for $P e_{p}>10$.

The noticeable disparity on the predictions of the total dispersion tensor components and of $v_{\text {eff, } x}$ between the two versions of the upscaled models may suggest that such errors impact the simplified upscaled model performance. However, it is worth recalling that the simplified upscaled model was obtained by comparing the orders of magnitude of the different terms in the complete upscaled model but not of the different terms that compose the coefficients themselves. Therefore, it is not surprising that some discrepancies on the 


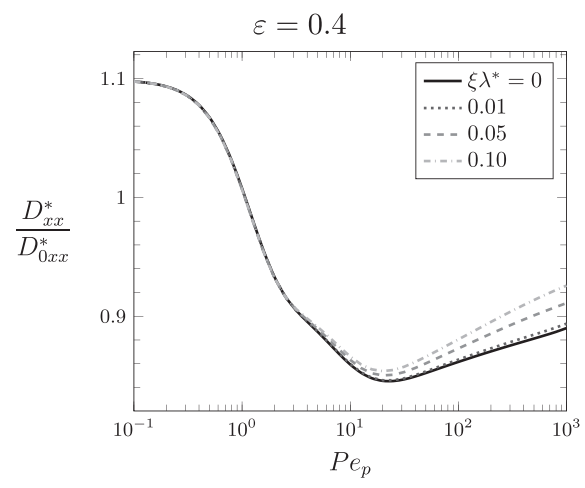

a)

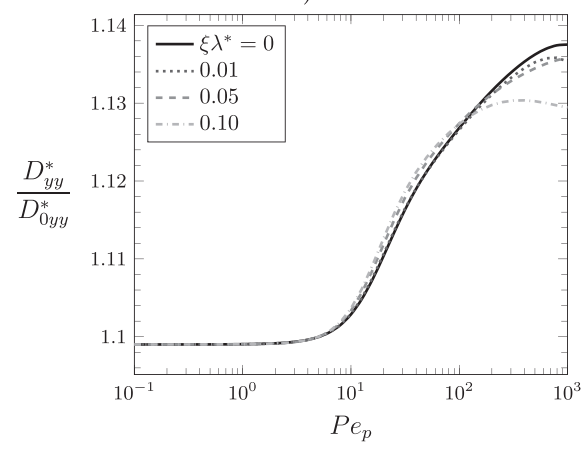

c)

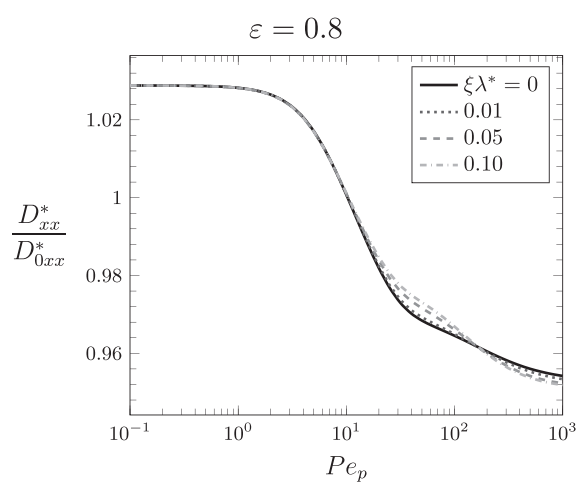

b)

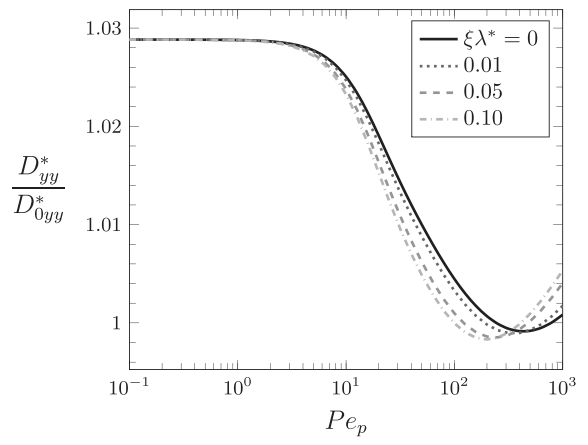

d)

Figure 5. Longitudinal ( $a$ and $b$ ) and transverse ( $c$ and d) components of the total dispersion tensor in the complete upscaled model with respect to the corresponding components in the simplified upscaled model versus the particle Péclet number $P e_{p}$ for four values of the cell Knudsen number $\lambda^{*}$. The predictions correspond to $\phi_{c}^{2}=1.0$ and porosity values of 0.4 (a and $c$ ) and $0.8(b$ and $d)$.

predictions of the coefficients arise, albeit such differences may not disqualify the predictive capabilities of the simplified upscaled model as confirmed by the analysis in section A2 of Appendix A.

Finally, in Figure 6, the predictions of the effective reaction rate coefficient (which is involved in both the complete and simplified upscaled models) are presented versus $P e_{p}$ for the same conditions as those considered for Figures 3 and 4 . These results indicate that $k_{\text {eff }}$ decreases as the porosity is increased in the simple geometry considered here. This is in agreement with the results presented in Figure 4 of the work by Valdés-Parada et al. (2017). Moreover, increasing the interfacial slip translates into an increment of $k_{\text {eff }}$ for

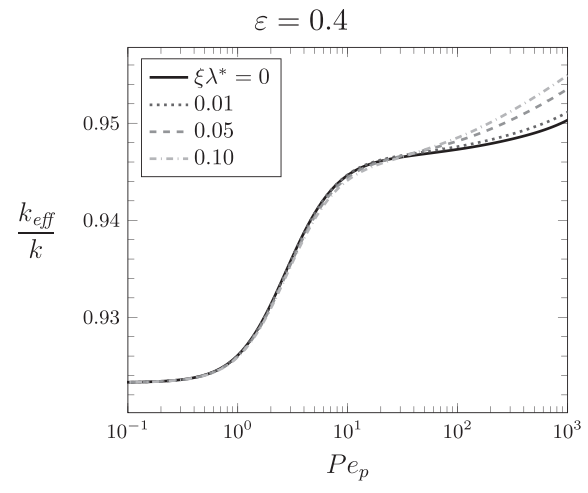

a)

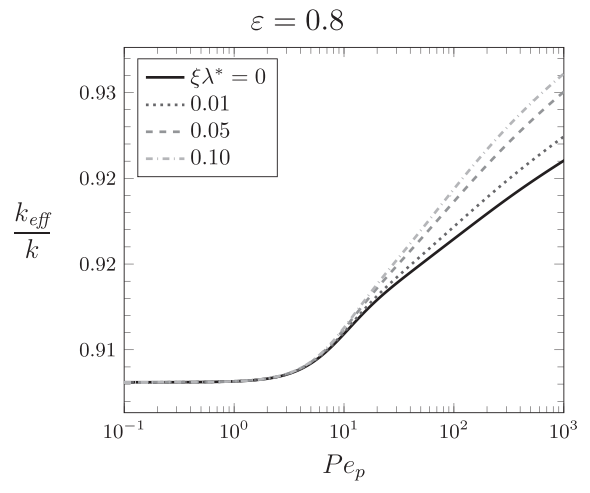

b)

Figure 6. Dimensionless effective reaction rate coefficient versus the particle Péclet number $P e_{p}$ for $\phi_{c}^{2}=1$ and four values of the cell Knudsen number $\lambda^{*}$. (a) $\varepsilon=0.4$, (b) $\varepsilon=0.8$. 


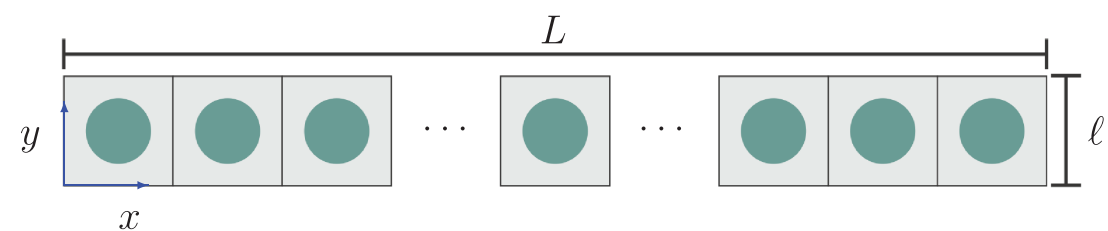

Figure 7. Sketch of the computational domain for the 2D system used to perform the direct numerical simulations. The length of the porous medium is chosen to be $L=100 \ell$.

large enough values of $P e_{p}$ (say $P e_{p}>10$ ). This can be explained by the fact that slip flow tends to improve convection at the pore scale, in particular close to the solid-fluid interface.

\subsection{Comparisons with Pore-Scale Numerical Simulations}

The performance of the simplified upscaled model is now compared to its complete version and to numerical simulations carried out at the pore scale. A model structure was used for the porous medium made of a square pattern of parallel cylinders having a square cross section considering the flow orthogonal to the cylinder axes and oriented along a principal direction of the pattern. The simulation domain of length $L$, including 100 square unit cells (of size $\ell$ ) in the flow direction ( $x$-direction), is the one sketched in Figure 7, assuming periodicity in the $y$-direction. The pore-scale equations are formulated in terms of the dimensionless velocity and pressure, respectively defined as $\mu /\left(\ell^{2}\left\|\nabla\langle p\rangle^{\beta}\right\|\right) \mathbf{v}$ and $p /\left(\ell\left\|\nabla\langle p\rangle^{\beta}\right\|\right)$. In addition, the dimensionless concentration and time are defined as $c^{*}=c / c_{\text {ref }}$ and $t^{*}=t \mathscr{D} / \ell^{2}$, respectively. Finally, coordinates are made dimensionless using $\ell$ as the reference length. In this analysis, $c_{r e f}$ is taken to be the inlet concentration (i.e., at $x^{*}=0$ ), which is assumed to be known and constant. A symmetry condition is imposed at the exit of the system (i.e., at $x^{*}=L / \ell$ ), and the initial species concentration is assumed to be zero in the entire domain. In summary, the following set of boundary and initial conditions are imposed:

$$
\text { at } x^{*}=0, \quad c^{*}=1,
$$

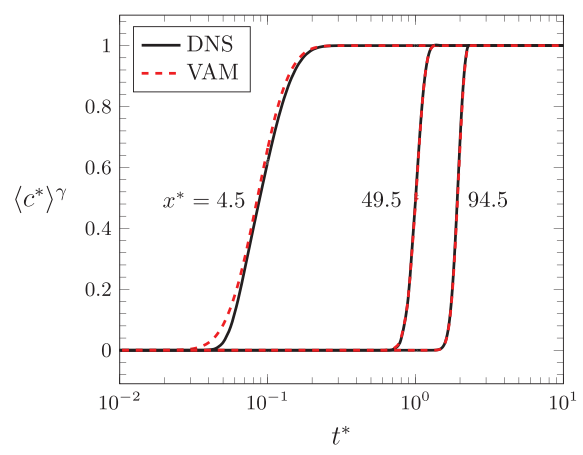

a)

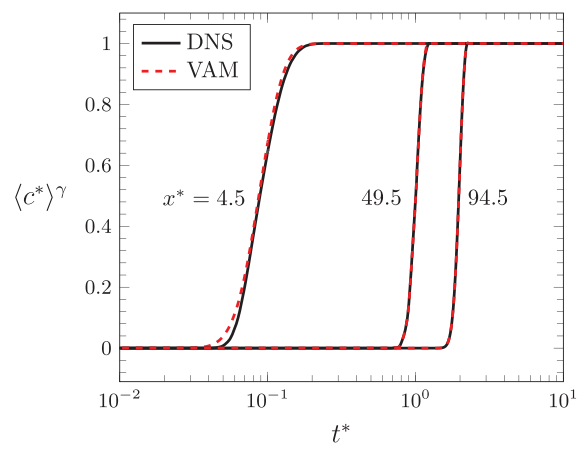

c)

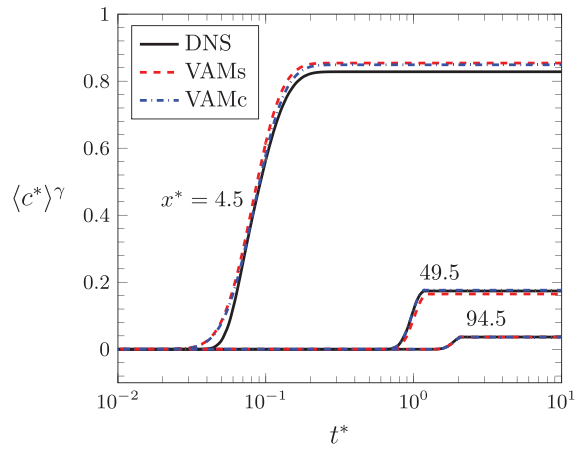

b)

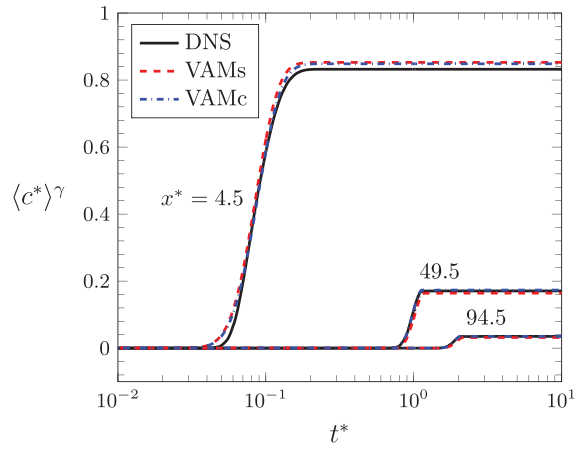

d)

Figure 8. Comparison of the dynamics of the intrinsic average concentration taken at particular positions of the macroscopic domain sketched in Figure 7. Concentration profiles result from DNS and the volume averaging method (VAM) including its simplified (VAMs) and complete (VAMc) versions for $P e_{p}=100, \varepsilon=0.8$ taking (a) $\xi \lambda^{*}=0, \phi_{c}^{2}=0$, (b) $\xi \lambda^{*}=0, \phi_{c}^{2}=1$, (c) $\xi \lambda^{*}=0.1, \phi_{c}^{2}=0$, and (d) $\xi \lambda^{*}=0.1, \phi_{c}^{2}=1$. 


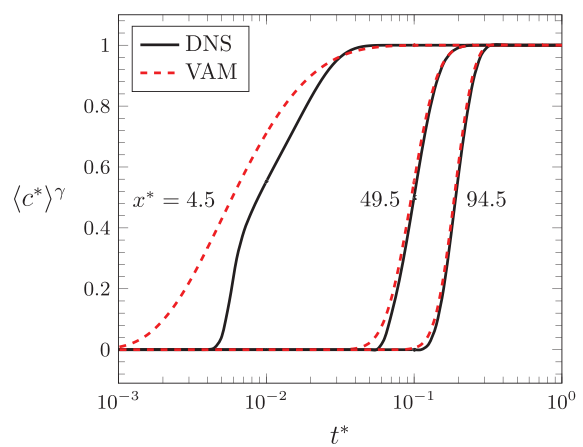

a)

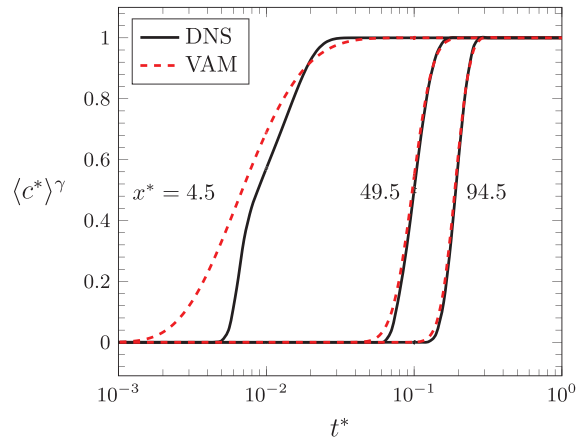

c)

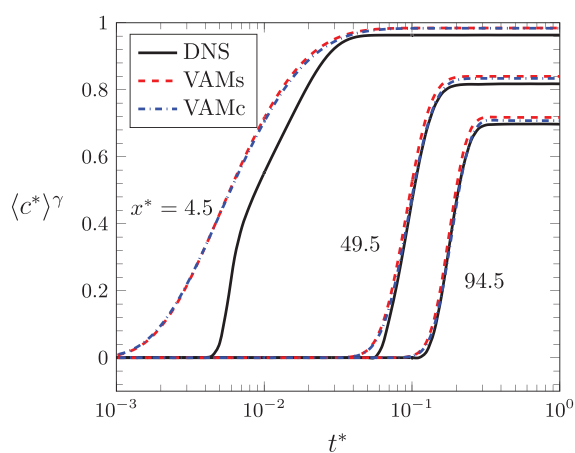

b)

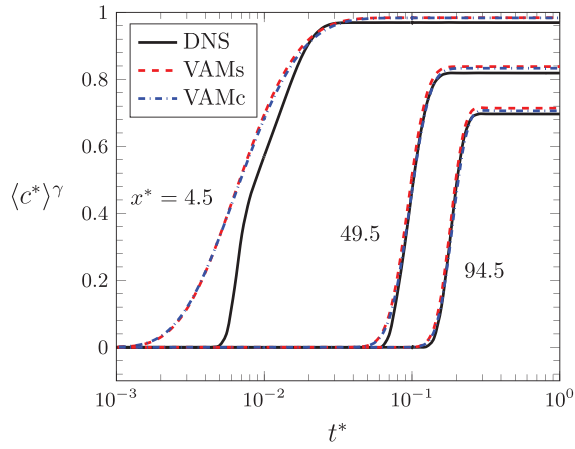

d)

Figure 9. Comparison of the dynamics of the intrinsic average concentration taken at particular positions of the system sketched in Figure 7 resulting from DNS and the volume averaging method (VAM) including its simplified (VAMs) and complete (VAMc) versions for $P e_{p}=1,000, \varepsilon=0.8$ taking (a) $\xi \lambda^{*}=0, \phi_{c}^{2}=0$, (b) $\xi \lambda^{*}=0, \phi_{c}^{2}=1$, (c) $\xi \lambda^{*}=0.1, \phi_{c}^{2}=0$, and (d) $\xi \lambda^{*}=0.1, \phi_{c}^{2}=1$.

$$
\begin{gathered}
\text { at } x^{*}=\frac{L}{\ell}, \quad \frac{\partial c^{*}}{\partial x^{*}}=0, \\
c^{*}\left(x^{*}, 0, t^{*}\right)=c^{*}\left(x^{*}, 1, t^{*}\right), \\
\text { when } t^{*}=0, \quad c^{*}=0 .
\end{gathered}
$$

Equivalent conditions are adopted to complete the formulation of the macroscale models. In addition, the solution of the dimensionless versions of the total mass and momentum transport equations at the pore scale is carried out in such a way that each unit cell is subject to a unitary pressure gradient as required by the dimensionless formulation.

The performance of the macroscale models is evaluated by analyzing the dynamics of the average concentration computed at particular points of the porous medium. More specifically, the sampling positions were chosen near the entrance $\left(x^{*}=4.5\right)$, near the middle $\left(x^{*}=49.5\right)$, and near the exit $\left(x^{*}=94.5\right)$ of the macroscopic domain. The resulting predictions are reported in Figures 8 and 9, taking $\varepsilon=0.8, P e_{p}=100$ and 1,000, respectively, under passive $\left(\phi_{c}^{2}=0\right)$ and reactive $\left(\phi_{c}^{2}=1\right)$ conditions as well as under the no-slip $\left(\xi \lambda^{*}=0\right)$ and slip flow condition $\left(\xi \lambda^{*}=0.1\right)$. The predictions from the upscaled models are provided in terms of both its complete $\left(\mathrm{VAM}_{c}\right)$ and simplified $\left(\mathrm{VAM}_{s}\right)$ versions. However, when no reaction takes place, both versions are identical (denoted simply as VAM). As shown in these figures, the agreement between the upscaled models and the DNS is excellent. Indeed, the relative error with respect to DNS remains below 5\% for situations in which the temporal (see constraint (12)) and length-scale constraints (see constraint (2)) that support the theory are met. In fact, it is only near the entrance zone and under highly convective transport (i.e., for $\left.P e_{p}=1,000\right)$ conditions that the largest differences are found. Furthermore, for this transport situation, the improvement provided by the complete upscaled model is barely noticeable, confirming the relevance of the 
simplified upscaled model for the reactive transport conditions considered here. At this point, it is worth noting that for $P e_{p}=1,000$, the ratio $\ell_{\gamma}(1+P e) / L=0.273$ and 1.221 for $\varepsilon=0.4$ and 0.8 , respectively. This indicates that the constraint $\ell_{\gamma} P e / L \ll 1$ is not met for $\varepsilon=0.8$. Nevertheless, the simplified model does not exhibit considerable differences with respect to the complete model, suggesting again that such constraint may be overly severe as noted in previous sections. Finally, it is pertinent to recall the temporal constraint given in (12). According to this constraint, the time scale at which the quasi-steady assumption is applicable is smaller than the characteristic diffusion time scale for which $t^{*}=1$ and decreases as the Péclet number is increased. This explains why there is agreement, in general, between the concentration profiles obtained from the macroscopic models and the DNS for $t^{*}>10^{-1}$ (Figure 8 for $P e_{p}=100$ ) or even at earlier times when $P e_{p}=1,000$ (Figure 9).

\section{Conclusions}

In this work, the problem of mass transport undergoing hydrodynamic slip (i.e., for a Knudsen number smaller than 0.1) and heterogeneous reaction in homogeneous porous media is analyzed with an upscaling approach. The pore-scale model was spatially smoothed using the volume averaging method giving rise to a macroscopic effective model involving three effective coefficients, namely, the total dispersion tensor, the effective convective velocity, and the effective reaction rate coefficient. This complete upscaled model is simplified on the basis of order of magnitude estimates and expansions in terms of the Kinetic and Péclet numbers. No simplification for the macroscopic mass transport equation is found from an expansion in terms of the Knudsen number. The effective convective velocity and total dispersion tensor involved in the simplified upscaled model correspond to those in the complete upscaled model under nonreactive conditions. This represents a net advantage in practice since the closure problems yielding these effective coefficients need to be solved only once regardless the value of the Kinetic number for given values of the Péclet and Knudsen numbers. The simplified model is found to be subject to the constraint $\ell_{\gamma} P e / L \ll 1$.

Predictions of the effective coefficients present in both the complete and simplified upscaled models are investigated versus the Péclet, Kinetic, and Knudsen numbers by solving the ancillary closure problems on a model 2D structure for two porosity values. Results show that, for large enough Péclet numbers ( $\gtrsim 10)$, the effective velocity, the transverse dispersion, and effective reaction rate coefficients increase with the Kinetic number. The opposite behavior is obtained for the longitudinal dispersion coefficient. These effects are enhanced as slip effects are more pronounced. These findings are compliant with the fact that slip flow smooths the pore-scale velocity (and consequently decreases the spatial deviations of the velocity), thus enhancing pore-scale convection. However, a more thorough analysis about the role of the microstructure on the prediction of the effective coefficients is certainly desirable. The macroscopic models and the effective coefficients are in agreement with those reported by Edwards et al. (1993) and Mauri (1991) who considered no-slip conditions. In addition, this work extents the previous study by Valdés-Parada et al. (2017) to convective transport within the context of slip flow.

Although noticeable differences are observed between the effective coefficients involved in the simplified and complete upscaled models, the dynamics of the average concentration fields are accurately described by the simplified upscaled model as assessed by comparison with pore-scale direct numerical simulations. The excellent performance of the simplified upscaled model is observed for conditions in which the temporal and spatial constraints imposed in the closure process are satisfied. In addition, for the simple structure under consideration, the simplified model is found to perform satisfactorily even for situations in which the constraint $\ell_{\gamma} P e / L \ll 1$ is not met, thus suggesting that this constraint is overly severe. Nevertheless, experimental investigations, as well as detailed pore-scale analyses, could certainly serve as a confirmation of these conclusions.

\section{Appendix A: Order of Magnitude Estimates of Closure Variables and Effective Coefficients}

The purpose of this section is to provide orders of magnitude estimates of the closure variables $\mathbf{b}$ and $s^{\prime}$, which are required for the derivation of the simplified upscaled model. To this end, consider a function, $\psi$, defined in the fluid phase. Its order of magnitude on the support $\Omega_{\gamma}$, of measure $\left|\Omega_{\gamma}\right|$, is defined as follows 
(Whitaker, 1983):

$$
\mathcal{O}(\psi)=\frac{1}{\left|\Omega_{\gamma}\right|} \int_{\Omega_{\gamma}}\|\psi\| d V
$$

An order of magnitude estimate of $\psi$, denoted by $\mathbf{O}(\psi)$, consists in finding a reasonable approximation of the above definition. In this regard, the derivations from Whitaker (1983) can be used to propose the following estimates for the gradient and the Laplacian of $\psi$ :

$$
\nabla \psi=\mathbf{O}\left(\frac{\psi}{\ell_{\gamma}}\right) ; \quad \nabla^{2} \psi=\mathbf{O}\left(\frac{\psi}{\ell_{\gamma}^{2}}\right) .
$$

If instead of considering a pore-scale variable, the interest lies upon estimating the derivative of a volume-averaged quantity, then the characteristic length $L$ should be used instead of $\ell_{\gamma}$ (Whitaker, 1999b).

\section{A1. Order of Magnitude Estimates of Closure Variables $b$ and $\boldsymbol{s}^{\prime}$}

On the basis of the above, one may estimate the orders of magnitude of closure variables from the corresponding closure problems in the bulk of phases or at interfaces by matching volume or surface source terms with each term involved in the differential equations or boundary conditions, respectively. More particularly, the interest here lies on estimating the order of magnitude of the closure variables $\mathbf{b}$ at the solid-fluid interface and $s^{\prime}$ both in the bulk of the fluid phase and at the fluid-solid interface. This is motivated by the fact that the terms in the effective coefficients $\mathbf{v}_{\text {eff }}$ and $k_{\text {eff }}$ for which an order of magnitude estimate is ultimately sought involve an area integral of $\mathbf{b}$ over $\mathscr{A}_{\gamma \kappa}$ and both volume and area integrals of $s^{\prime}$ over $\mathscr{V}_{\gamma}$ and $\mathscr{A}_{\gamma \kappa}$, respectively.

The order of magnitude estimate for $\mathbf{b}$ at $\mathscr{A}_{\gamma \kappa}$ is carried out on Problem I, which is given by

$$
\begin{gathered}
\tilde{\mathbf{v}}+\mathbf{v} \cdot \nabla \mathbf{b}=\mathscr{D} \nabla^{2} \mathbf{b}+\frac{k}{V_{\gamma}} \int_{\mathscr{A}_{\gamma \kappa}} \mathbf{b} \quad d A, \quad \text { in the } \gamma \text {-phase, } \\
-\mathbf{n} \cdot(\mathscr{D} \nabla \mathbf{b})-k \mathbf{b}=\mathbf{n} \mathscr{D}, \quad \text { at the } \gamma-\kappa \text { interface } \\
\mathbf{b}\left(\mathbf{r}+\mathbf{l}_{i}\right)=\mathbf{b}(\mathbf{r}), \quad i=1,2,3 \\
\langle\mathbf{b}\rangle^{\gamma}=\mathbf{0} .
\end{gathered}
$$

A volume and a surface source ( $\tilde{\mathbf{v}}$ and $\mathbf{n} \mathscr{D}$ in equations (A3a) and (A3b), respectively) on which $\mathbf{b}$ is expected to depend are to be considered. The analysis of the impact of the volume source may be simplified by a simple algebraic manipulation on the last term on the right-hand side of equation (A3a). The surface integration of the boundary condition in equation (A3b) over $\mathscr{A}_{\gamma \kappa}$, followed by division by $V_{\gamma}$, yields

$$
\frac{k}{V_{\gamma}} \int_{\mathscr{A}_{\gamma \kappa}} \mathbf{b} \quad d A=-\frac{\mathscr{D}}{V_{\gamma}} \int_{\mathscr{A}_{\gamma \kappa}} \mathbf{n} \cdot \nabla \mathbf{b} \quad d A-\frac{\mathscr{D}}{V_{\gamma}} \int_{\mathscr{A}_{\gamma \kappa}} \mathbf{n} d A .
$$

Turning the attention to the right-hand side of this last equation, it is evident that due to the homogeneity of the medium, the last term is zero. Using the averaging theorem and the fact that $\nabla \mathbf{b}$ is periodic allows to show that the first term is equal to $-\mathscr{D}\left\langle\nabla^{2} \mathbf{b}\right\rangle^{\gamma}$. Using this result in equation (A3a) leads to

$$
\tilde{\mathbf{v}}+\mathbf{v} \cdot \nabla \mathbf{b}=\mathscr{D} \nabla^{2} \mathbf{b}-\mathscr{D}\left\langle\nabla^{2} \mathbf{b}\right\rangle^{\gamma}, \quad \text { in the } \gamma \text {-phase, }
$$

in which both terms on the right hand side have the same order of magnitude. From this form of the balance equation for $\mathbf{b}$, the influence of the volume source, $\tilde{\mathbf{v}}$, on $\mathbf{b}$ at $\mathscr{A}_{\gamma \kappa}$ may now be analyzed by considering this equation at $\mathscr{A}_{\gamma \kappa}$, which remains formally unchanged in the presence of slip. However, it is important to notice that the source, $\tilde{\mathbf{v}}$, is maximum in the no-slip case, so that $\mathbf{b}$ is also the largest in magnitude in that case. A numerical illustration of this will be provided below. With the purpose of deriving an upper 


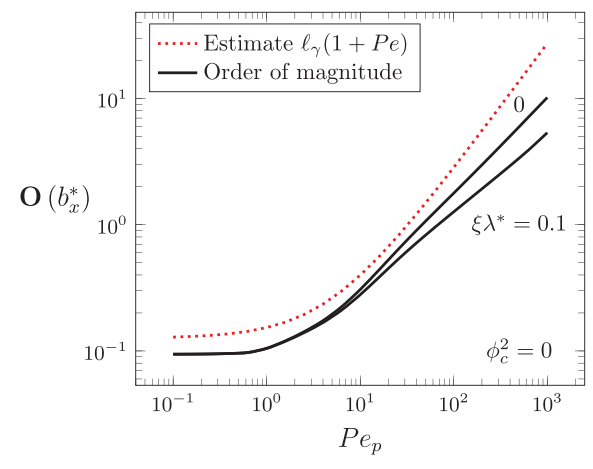

a)

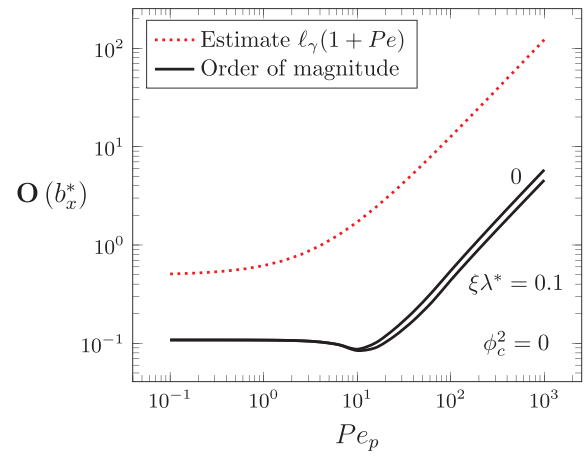

c)

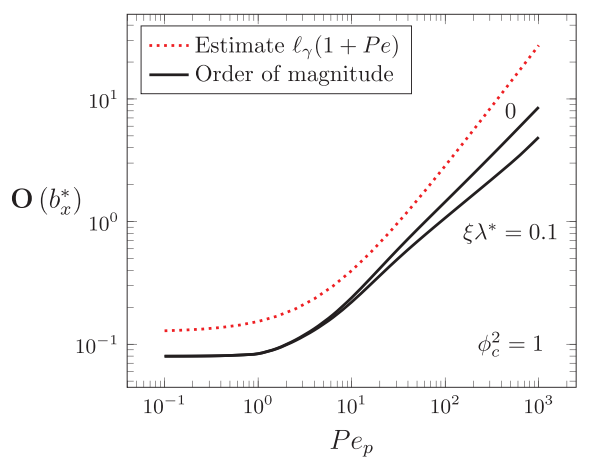

b)

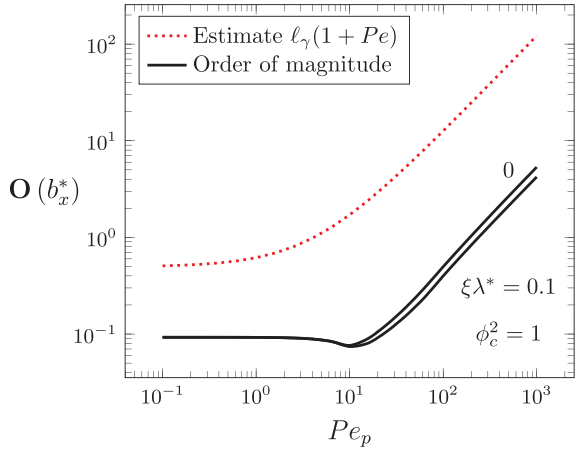

d)

Figure A1. Comparison between the estimate and the actual order of magnitude of the $x$-component of closure variable $\mathbf{b}$ at $\mathscr{A}_{\gamma \kappa}$ normalized by the size of the unit cell, $\ell$, for (a) $\varepsilon=0.4, \phi_{c}^{2}=0$, (b) $\varepsilon=0.4, \phi_{c}^{2}=1$, (c) $\varepsilon=0.8$, $\phi_{c}^{2}=0$, and (d) $\varepsilon=0.8, \phi_{c}^{2}=1$. In each case, the influence of the interfacial slip is exhibited with results obtained with $\xi \lambda^{*}=0$ and $\xi \lambda^{*}=0.1$.

bound for $\mathbf{O}(\mathbf{b})$, the analysis can hence be safely carried out in the no-slip case. Under these circumstances, equation (A5) allows to write

$$
-\langle\mathbf{v}\rangle^{\gamma}=\mathscr{D} \nabla^{2} \mathbf{b}-\mathscr{D}\left\langle\nabla^{2} \mathbf{b}\right\rangle^{\gamma}, \quad \text { at the } \gamma-\kappa \text { interface, }
$$

from which an order of magnitude for $\mathbf{b}$ at $\mathscr{A}_{\gamma \kappa}$ can be obtained as

$$
\mathbf{b}=\mathbf{O}\left(\frac{v \ell_{\gamma}^{2}}{\mathscr{D}}\right)=\mathbf{O}\left(\ell_{\gamma} P e\right)
$$

From the boundary condition in equation (A3b), an alternative order of magnitude estimate can be derived by matching the two terms on the left hand side to the source to give

$$
\mathbf{b}=\mathbf{O}\left(\frac{\mathscr{D}}{\mathscr{D} / \ell_{\gamma}+k}\right)=\mathbf{O}\left(\ell_{\gamma} \frac{1}{1+\phi^{2}}\right)=\mathbf{O}\left(\ell_{\gamma}\right) .
$$

The latter relationship is the leading order of magnitude estimate for $\mathbf{b}$ when $P e \leq 1$ and $\phi^{2}<1$, whereas equation (A7) can be considered as the appropriate one when $P e>1$. This leads to propose the following order of magnitude estimate for $\mathbf{b}$ at $\mathscr{A}_{\gamma \kappa}$ as

$$
\mathbf{b}=\mathbf{O}\left(\ell_{\gamma}(1+P e)\right),
$$

which may be used for any value of $P e$.

In order to verify the relevance of this estimate, a numerical evaluation of the order of magnitude of the $x$-component of $\mathbf{b}$ at $\mathscr{A}_{\gamma \kappa}$ was carried out. This was performed on the unit cell represented in Figure 2 for $\varepsilon=0.4$ and $\varepsilon=0.8$, for two values of $\xi K n$ corresponding to $\xi \lambda^{*}=0$ and $\xi \lambda^{*}=0.1\left(\lambda^{*}=K n \ell_{\gamma} / \ell\right)$, two values of $\phi^{2}$ corresponding to $\phi_{c}^{2}=0$ and $\phi_{c}^{2}=1\left(\phi_{c}^{2}=\phi^{2} \ell / \ell_{\gamma}\right)$, and $P e_{p}$ ranging between 0.1 and $10^{3}$ 


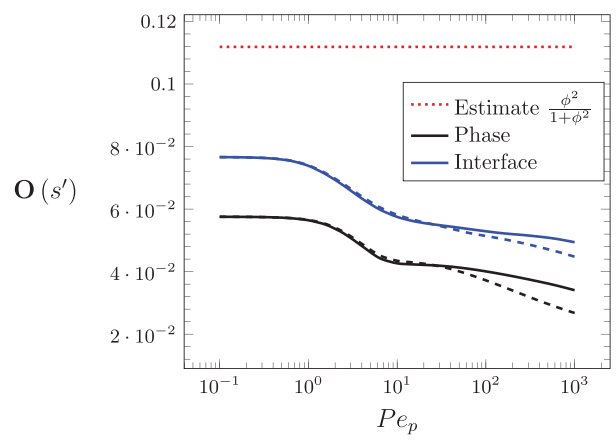

a)

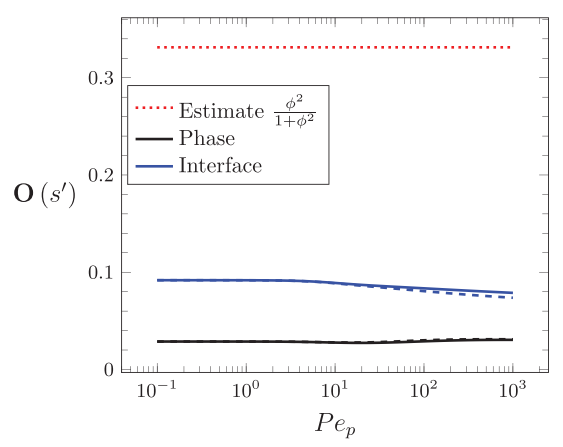

b)

Figure A2. Comparisons between the estimate given in equation (A13) and the order of magnitude of closure variable $s^{\prime}$ computed at $\mathscr{A}_{\gamma \kappa}$ and in $V_{\gamma}$. (a) $\varepsilon=0.4$; (b) $\varepsilon=0.8$. In both cases, $\phi_{c}^{2}=1$; in addition, the solid lines correspond to $\xi \lambda^{*}=0$ and the dashed lines to $\xi \lambda^{*}=0.1$.

$\left(P e_{p}=P e \ell_{\kappa} \varepsilon /\left(\ell_{\gamma}(1-\varepsilon)\right)\right)$. Results of $b_{x}^{*}=b_{x} / \ell(\ell$ is the size of the unit cell) are represented in Figure A1 together with the order of magnitude provided in equation (A9).

This figure shows that this order of magnitude estimate, although being an overestimate, reproduces the actual tendency of the numerical results. Moreover, it also confirms that the order of magnitude of $\mathbf{b}$ increases when slip effects decrease as already mentioned, justifying the use of the no-slip condition for the derivation of its estimate.

At this point, attention can be focused on the closure variable $s^{\prime}$, solution of closure Problem II given by

$$
\begin{gathered}
\mathbf{v} \cdot \nabla s^{\prime}=\mathscr{D} \nabla^{2} s^{\prime}+\frac{k}{V_{\gamma}} \int_{\mathscr{A}_{\gamma \kappa}} s^{\prime} \quad d A+\frac{k A_{\gamma \kappa}}{V_{\gamma}}, \quad \text { in the } \gamma \text {-phase, } \\
-\mathbf{n} \cdot\left(\mathscr{D} \nabla s^{\prime}\right)-k s^{\prime}=k, \quad \text { at the } \gamma-\kappa \text { interface, } \\
s^{\prime}\left(\mathbf{r}+\mathbf{l}_{i}\right)=s^{\prime}(\mathbf{r}), \quad i=1,2,3, \\
\left\langle s^{\prime}\right\rangle^{\gamma}=0 .
\end{gathered}
$$

Performing the area integral of equation (A10b) over $\mathscr{A}_{\gamma \kappa}$, dividing the result by $V_{\gamma}$, employing the averaging theorem, and taking into account that $\nabla s^{\prime}$ is a periodic field yield

$$
\frac{k}{V_{\gamma}} \int_{\mathscr{A}_{\gamma \kappa}} s^{\prime} \quad d A=-\frac{k a_{v}}{\varepsilon}-\mathscr{D}\left\langle\nabla^{2} s^{\prime}\right\rangle^{\gamma} .
$$

When this result is substituted back into equation (A10a), it gives

$$
\mathbf{v} \cdot \nabla s^{\prime}=\mathscr{D} \nabla^{2} s^{\prime}-\mathscr{D}\left\langle\nabla^{2} s^{\prime}\right\rangle^{\gamma},
$$

showing that there is no volume source for this problem. As a consequence, the order of magnitude estimate can be extracted from the boundary condition in equation (A10b). When both the diffusive and reactive flux terms in this equation are matched to the source, $k$, this gives

$$
s^{\prime}=\mathbf{O}\left(\frac{\phi^{2}}{1+\phi^{2}}\right) \text {. }
$$

A comparison of this estimate with the actual order of magnitude obtained from the solution of Problem II and computed at the interface $\mathscr{A}_{\gamma \kappa}$ and in $V_{\gamma}$ is presented in Figure A2 versus $P e_{p}$ for $\varepsilon=0.4$ and $\varepsilon=0.8$, taking $\phi_{c}^{2}=1, \xi \lambda^{*}=0$, and $\xi \lambda^{*}=0.1$, considering the unit cell of Figure 2.

The results in Figure A2 show that the order of magnitude estimate in equation (A13) is a reasonable one. Since $\phi^{2}<1$, the above estimate can be simplified to

$$
s^{\prime}=\mathbf{O}\left(\phi^{2}\right),
$$

which is an overestimate, whereas an obvious underestimate is $s^{\prime}=0$. 


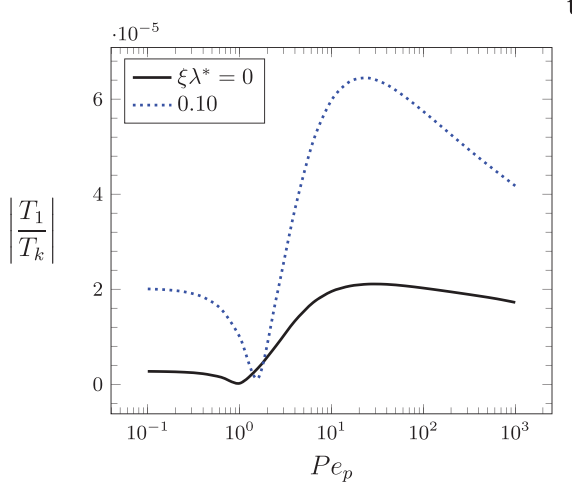

a)

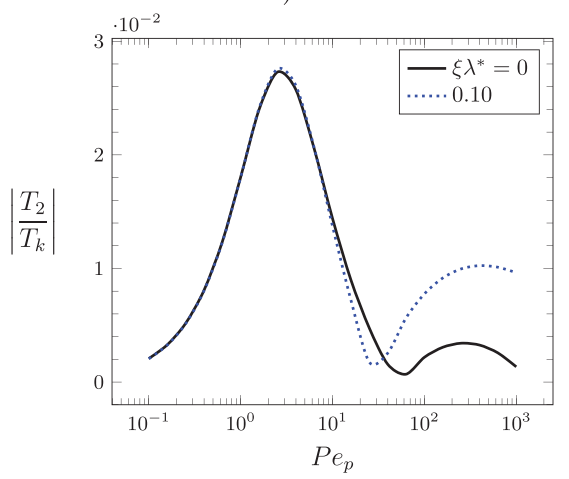

c)

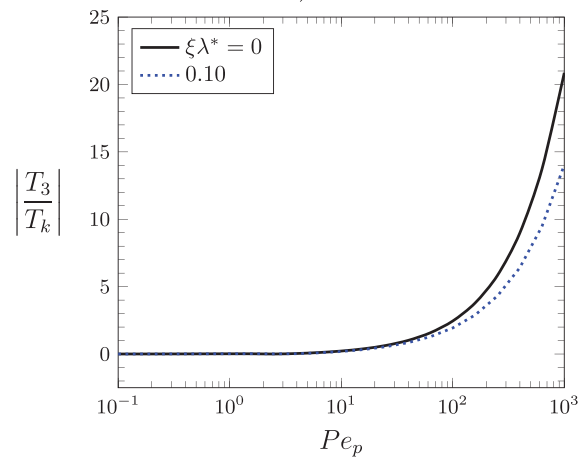

e) t!

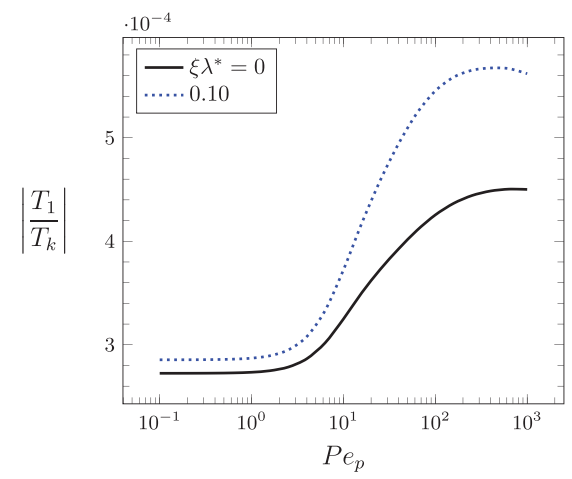

b)

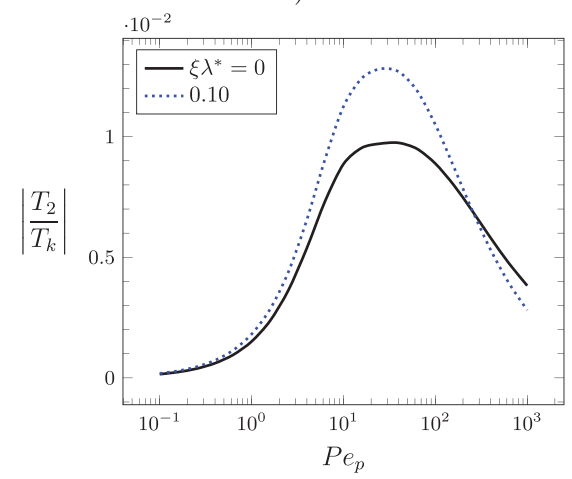

d)

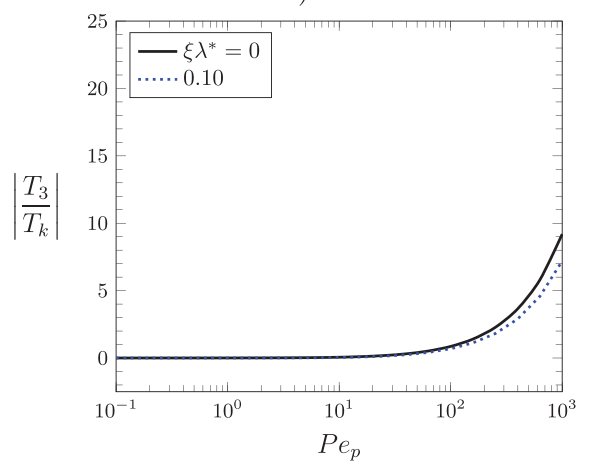

f)

Figure A3. Absolute values of the ratios between the reactive contributions to $\mathbf{v}_{\text {eff }}$ with respect to $k_{\text {eff }} \varepsilon^{-1}$ for (a), (c), and (e) $\varepsilon=0.4$; (b), (d), and (f) $\varepsilon=0.8 . \phi_{c}^{2}=1 . T_{1}=\left\langle v_{x} s^{\prime}\right\rangle^{\gamma}, T_{2}=\frac{\mathscr{D}}{V_{\gamma}} \int_{\mathscr{A}_{\gamma \kappa}} n_{x} s^{\prime} d A, T_{3}=\frac{k}{V_{\gamma}} \int_{\mathscr{A}_{\gamma \kappa}} b_{x} d A$, and $T_{k}=\frac{k}{\varepsilon A_{\gamma \kappa}} \int_{\mathscr{A}_{\gamma \kappa}}\left(1+s^{\prime}\right) \quad d A$.

\section{A2. Comparison of the Convective-Like and Reactive Terms in the Macroscopic Model}

From the above estimates on $\mathbf{b}$ and $s^{\prime}$, a comparative analysis of the orders of magnitude of the convective-like terms $\left\langle\mathbf{v} s^{\prime}\right\rangle^{\gamma} \cdot \nabla\langle c\rangle^{\gamma},\left(\frac{\mathscr{D}}{V_{\gamma}} \int_{\mathscr{A}_{\gamma \kappa}} \mathbf{n} s^{\prime} d A\right) \cdot \nabla\langle c\rangle^{\gamma}$, and $\left(\frac{k}{V_{\gamma}} \int_{\mathscr{A}_{\gamma \kappa}} \mathbf{b} d A\right) \cdot \nabla\langle c\rangle^{\gamma}$ with respect to the reactive term $\frac{k a_{v}}{\varepsilon} \frac{1}{A_{\gamma \kappa}} \int_{\mathscr{A}_{\gamma \kappa}}\left(s^{\prime}+1\right) \quad d A\langle c\rangle^{\gamma}$ is proposed in section 5.1. The objective here is to verify that the simplifications of the macroscopic model resulting from these comparisons are justified. This is performed for the unit cell represented in Figure 2. Since the macroscopic length-scale, $L$, at which $\langle c\rangle^{\gamma}$ is expected to experience significant variations is not specified at this point, the verification is carried out by computing the ratios $\left|\frac{T_{i}}{T_{k}}\right|$, where $T_{i}$ is given by $T_{1}=\left\langle v_{x} s^{\prime}\right\rangle^{\gamma}, T_{2}=\frac{\mathscr{D}}{V_{\gamma}} \int_{\mathscr{A}_{\gamma \kappa}} n_{x} s^{\prime} d A$, and $T_{3}=\frac{k}{V_{\gamma}} \int_{\mathscr{A}_{\gamma \kappa}} b_{x} d A$, whereas 
$T_{k}=\frac{k}{\varepsilon A_{\gamma \kappa}} \int_{\mathscr{A}_{\gamma \kappa}}\left(1+s^{\prime}\right) \quad d A$. Since $a_{v}=\mathbf{O}\left(\ell_{\gamma}^{-1}\right)$, these ratios must be further multiplied by $\ell_{\gamma} / L$ to quantify the magnitude of the corresponding convective-like terms relative to the reactive term in the macroscopic model. The ratios $\left|\frac{T_{i}}{T_{k}}\right|$ are represented versus $P e_{p}$ in Figure A3 for $\varepsilon=0.4$ and $\varepsilon=0.8, \phi_{c}^{2}=1$ and, in each case, for $\xi \lambda^{*}=0$ and $\xi \lambda^{*}=0.1$.

Although the order of magnitude analysis reported in section 5.1 indicates that the constraint $\varepsilon \frac{\ell_{\gamma}}{L} P e \ll 1$ is necessary for $\left\langle\mathbf{v} s^{\prime}\right\rangle^{\gamma} \cdot \nabla\langle c\rangle^{\gamma} \ll \frac{k a_{v}}{\varepsilon} \frac{1}{A_{\gamma \kappa}} \int_{\mathscr{A}_{\gamma \kappa}}\left(s^{\prime}+1\right) \quad d A\langle c\rangle^{\gamma}$ to hold, results in Figures A3a and A3b indicate that this remains valid whatever the Péclet number. Numerical results obtained on the unit cell of many other model 2D structures lead to the same conclusion, suggesting that this constraint is indeed much too restrictive. This can be explained by the fact that the order of magnitude estimate $\left\langle\mathbf{v} s^{\prime}\right\rangle^{\gamma}=\mathbf{O}\left(v \phi^{2}\right)$ is a strong overestimate. In the absence of more precise bounds, this constraint may however be kept to carry out the associated simplification of $\mathbf{v}_{\text {eff }}$.

From Figures A3c and A3d, it clearly appears that the simplification resulting from $\left(\frac{\mathscr{D}}{V_{\gamma}} \int_{\mathscr{A}_{\gamma \kappa}} \mathbf{n} s^{\prime} d A\right)$. $\nabla\langle c\rangle^{\gamma} \ll \frac{k a_{v}}{\varepsilon} \frac{1}{A_{\gamma \kappa}} \int_{\mathscr{A}_{\gamma \kappa}}\left(s^{\prime}+1\right) \quad d A \quad\langle c\rangle^{\gamma}$ whatever the Péclet number is a reasonable one, as obtained from the order of magnitude analysis. In addition, Figures A3e and A3f show that the ratio $\left|\frac{T_{3}}{T_{k}}\right|$ increases with $P e_{p}$, justifying the constraint $\frac{\ell_{\gamma}}{L}(1+P e) \ll 1$ obtained from the order of magnitude analysis for $\left(\frac{k}{V_{\gamma}} \int_{\mathscr{A}_{\gamma \kappa}} \mathbf{b} d A\right)$. $\nabla\langle c\rangle^{\gamma} \ll \frac{k a_{v}}{\varepsilon} \frac{1}{A_{\gamma \kappa}} \int_{\mathscr{A}_{\gamma \kappa}}\left(s^{\prime}+1\right) \quad d A \quad\langle c\rangle^{\gamma}$ to be valid.

From these results, it can be concluded that the simplifications carried out on the macroscopic model on the basis of the order of magnitude analysis are reasonable.

\section{References}

DL is thankful to Universidad Autónoma Metropolitana for the financial aid to carry out a research stay during November 2018, and FJVP is thankful to the Centre National de la Recherche Scientifique (CNRS) for allowing a research stay during the month of April 2019. The data used for the figures provided in this article are available at the following link with their names corresponding to the figure numbers: https://mycore.core-cloud. net/index.php/s/Pn3kean1RzI4wr1.
Adrover, A., Cerbelli, S., Garofalo, F., \& Giona, M. (2009). Convection-dominated dispersion regime in wide-bore chromatography: A transport-based approach to assess the occurrence of slip flows in microchannels. Analytical Chemistry, 81, 8009-8014. https://doi.org/ 10.1021/ac901504u

Alhashmi, Z., Blunt, M., \& Bijeljic, B. (2015). Predictions of dynamic changes in reaction rates as a consequence of incomplete mixing using pore scale reactive transport modeling on images of porous media. Journal of Contaminant Hydrology, 179, 171-181. https://doi. org/10.1016/j.jconhyd.2015.06.004

Altevogt, A., Rolston, D., \& Whitaker, S. (2003). New equations for binary gas transport in porous media, Part 1: Equation development. Advances in Water Resources, 26, 695-715. https://doi.org/10.1016/S0309-1708(03)00050-2

Arkilic, E. B., Breuer, K. S., \& Schmidt, M. A. (2001). Mass flow and tangential momentum accommodation in silicon micromachined channels. Journal of Fluid Mechanics, 437, 29-43. https://doi.org/10.1017/S0022112001004128

Auriault, J. L., Boutin, C., \& Geindreau, C. (2009). Homogenization of coupled phenomena in heterogenous media. Hoboken: ISTE LTD.

Battiato, I., \& Tartakovsky, D. M. (2011). Applicability regimes for macroscopic models of reactive transport in porous media. Journal of Contaminant Hydrology, 120-121, 18-26. https://doi.org/10.1016/j.jconhyd.2010.05.005

Battiato, I., Tartakovsky, D., Tartakovsky, A., \& Scheibe, T. (2009). On breakdown of macroscopic models of mixing-controlled heterogeneous reactions in porous media. Advances in Water Resources, 32(11), 1664-1673. https://doi.org/10.1016/j.advwatres.2009.08. 008

Bear, J. (2018). Modeling Phenomena of Flow and Transport in Porous Media, (theory and applications of transport in porous media). Cham, Switzerland: Springer.

Beauchamp, M., \& Schure, M. (2019). Simulation and theory of open-tube dispersion in short and long capillaries with slip boundaries and retention. Journal of Chromatography A, 1588, 85-98. https://doi.org/10.1016/j.chroma.2018.12.040

Boso, F., \& Battiato, I. (2013). Homogenizability conditions for multicomponent reactive transport. Advances in Water Resources, 62, 254-265. https://doi.org/10.1016/j.advwatres.2013.07.014

Bringedal, C., Berre, I., Pop, I. S., \& Radu, F. A. (2016). Upscaling of non-isothermal reactive porous media flow with changing porosity. Transport in Porous Media, 114(2), 371-393. https://doi.org/10.1007/s11242-015-0530-9

Dadvar, M., \& Sahimi, M. (2007). The effective diffusivities in porous media with and without nonlinear reactions. Chemical Engineering Science, 62, 1466-1476. https://doi.org/10.1016/j.ces.2006.12.002

Davit, Y., Bell, C. G., Byrne, H. M., Chapman, L. A., Kimpton, L. S., Lang, G. E., et al. (2013). Homogenization via formal multiscale asymptotics and volume averaging: How do the two techniques compare? Advances in Water Resources, 62, 178-206. https://doi.org/10. 1016/j.advwatres.2013.09.006

Edwards, D., Shapiro, M., \& Brenner, H. (1993). Dispersion and reaction in two-dimemsional model porous media. Phys. Fluids, 5, 837-848. https://doi.org/doi:10.1063/1.858631

Froment, G., Bischoff, K., \& Wilde, J. D. (2010). Chemical Reactor Analysis and Design (3rd ed.). Hoboken, NJ: Wiley.

Gao, J., Xing, H., Tian, Z., Pearce, J. K., Sedek, M., Golding, S. D., \& Rudolph, V. (2017). Reactive transport in porous media for CO sequestration: Pore scale modeling using the lattice Boltzmann method. Computers \& Geosciences, 98, 9-20. https://doi.org/10.1016/j. cageo.2016.09.008 
Gray, W. (1975). A derivation of the equations for multiphase transport. Chemical Engineering Science, 30, 229-233. https://doi.org/10. 1016/0009-2509(75)80010-8

Gray, W., \& Miller, C. (2014). Introduction to the Thermodynamically Constrained Averaging Theory for Porous Medium Systems. Cham: Springer. https://doi.org/10.1007/978-3-319-04010-3

Harley, J. C., Huang, Y., Bau, H. H., \& Zemel, J. N. (1995). Gas flow in micro-channels. Journal of Fluid Mechanics, 284, 257-274. https:// doi.org/10.1017/S0022112095000358

Howes, F., \& Whitaker, S. (1985). The spatial averaging theorem revisited. Chemical Engineering Science, 40, 1387-1392. https://doi.org/ 10.1016/0009-2509(85)80078-6

Lasseux, D., \& Valdés-Parada, F. J. (2017). Symmetry properties of macroscopic transport coefficients in porous media. Physics of Fluids, 29(4), 043303. https://doi.org/10.1063/1.4979907

Lasseux, D., Valdés-Parada, F., Ochoa-Tapia, J., \& Goyeau, B. (2014). A macroscopic model for slightly compressible gas slip-flow in homogeneous porous media. Physics of Fluids, 26, 053102. https://doi.org/https://doi.org/10.1063/1.4875812

Lasseux, D., Valdés-Parada, F., \& Porter, M. (2016). An improved macroscale model for gas slip flow in porous media. Journal of Fluid Mechanics, 805, 118-146. https://doi.org/10.1017/jfm.2016.562

Mauri, R. (1991). Dispersion, convection, and reaction in porous media. Physics of Fluids, 3, 743-756. https://doi.org/doi:10.1063/1.858007

Maxwell, J. C. (1879). On stresses in rarified gases arising from inequalities of temperature. Philosophical Transactions of the Royal Society of London, 170, 231-256. https://doi.org/10.1098/rstl.1879.0067

Niemi, A., Bear, J., \& Bensabat, J. (2017). Geological Storage of $\mathrm{CO}_{2}$ in Deep Saline Formations. Netherlands: Springer-Verlag GmbH. https:// doi.org/10.1007/978-94-024-0996-3

Nordbotten, J., \& Celia, M. (2011). Geological Storage of $\mathrm{CO}_{2}$ : Modeling Approaches for Large-Scale Simulation. Hoboken, NJ: John Wiley $\&$ Sons Inc.

Oya, S., \& Valocchi, A. (1998). Transport and biodegradation of solutes in stratified aquifers under enhanced in situ bioremediation conditions. Water Resources Research, 34(12), 3323-3334. https://doi.org/10.1029/98WR02770

Porta, G. M., Ceriotti, G., \& Thovert, J. F. (2016). Comparative assessment of continuum-scale models of bimolecular reactive transport in porous media under pre-asymptotic conditions. Journal of Contaminant Hydrology, 185-186, 1-13. https://doi.org/10.1016/j.jconhyd. 2015.12.003

Porta, G. M., Chaynikov, S., Thovert, J. F., Riva, M., Guadagnini, A., \& Adler, P. M. (2013). Numerical investigation of pore and continuum scale formulations of bimolecular reactive transport in porous media. Advances in Water Resources, 62, 243-253. https://doi.org/10.1016/ j.advwatres.2013.09.007

Scheibe, T., Perkins, W., Richmond, M., McKinley, M., Romero-Gomez, P. D. J., Oostrom, M., et al. (2015). Pore-scale and multiscale numerical simulation of flow and transport in a laboratory-scale column. Water Resources Research, 51, 1023-1035. https://doi.org/10. 1002/2014wr015959

Slattery, J. C. (1999). Advanced Transport Phenomena (Cambridge Series in Chemical Engineering). Cambridge: Cambridge University Press. www.cambridge.org/9780521635653

Smits, W., Deridder, S., \& Desmet, G. (2014). Theoretical study on the impact of slip flow on chromatographic performance. Journal of Chromatography A, 1366, 120-125. https://doi.org/10.1016/j.chroma.2014.09.027

Tian, Z., \& Wang, J. (2017). Lattice Boltzmann simulation of $\mathrm{CO}_{2}$ reactive transport in network fractured media. Water Resources Research, 53, 7366-7381. https://doi.org/10.1002/2017wr021063

Valdés-Parada, F., Aguilar-Madera, C., \& Alvarez-Ramírez, J. (2011). On diffusion, dispersion and reaction in porous media. Chemical Engineering Science, 66, 2177-2190. https://doi.org/10.1016/j.ces.2011.02.016

Valdés-Parada, F., Lasseux, D., \& Whitaker, S. (2017). Diffusion and heterogeneous reaction in porous media: The macroscale model revisited. International Journal of Chemical Reactor Engineering, 15(16), 20170151. https://doi.org/10.1515/ijcre-2017-0151

Whitaker, S. (1983). Fundamental Principles of Heat Transfer. New York: Krieger Publishing Company.

Whitaker, S. (1988). Levels of simplification: The use of assumptions, restrictions and constraints in engineering analysis. Chemical Engineering Education, 22, 104-108.

Whitaker, S. (1999a). Discontinuities in chemical engineering education. Chemical Engineering Education, 33, $18-25$.

Whitaker, S. (1999b). The Method of Volume Averaging. Netherlands: Kluwer Academic Publishers. https://doi.org/10.1007/978-94-0173389-2

Whitaker, S. (2009). Derivation and application of the Stefan-Maxwell equations. Revista Mexicana de Ingenieria Quimica, 18(3), 213-244.

Wood, B., \& Valdés-Parada, F. (2013). Volume averaging: Local and nonlocal closures using a Green's function approach. Advances in Water Resources, 51, 139-167. https://doi.org/10.1016/j.advwatres.2012.06.008

Yan, X., \& Li, N. (2017). Simulation of solute dispersion in particle packs by the volume averaging method. Computers \& Chemical Engineering, 98, 154-160. https://doi.org/10.1016/j.compchemeng.2016.12.021

Yan, X., \& Wang, Q. (2013). Numerical investigation into the effects of ordered particle packing and slip flow on the performance of chromatography. Journal of Separation Science, 36, 1524-1529. https://doi.org/10.1002/jssc.201201109

Yang, X., Mehmani, Y., Perkins, W. A., Pasquali, A., Schnherr, M., Kim, K., et al. (2016). Intercomparison of 3D pore-scale flow and solute transport simulation methods. Advances in Water Resources, 95, 176-189. https://doi.org/10.1016/j.advwatres.2015.09.015

Zhang, L., \& Seaton, N. (1994). The application of continuum equations to diffusion and reaction in pore networks. Chemical Engineering Science, 49, 41-50. https://doi.org/10.1016/0009-2509(94)85032-1 